\title{
Optimal and Cut-free Tableaux for Propositional Dynamic Logic with Converse
}

\author{
Rajeev Goré ${ }^{1}$ and Florian Widmann ${ }^{1,2}$ \\ Logic and Computation Group ${ }^{1}$ and NICTA $^{2 \star}$, The Australian National University \\ Canberra, ACT 0200, Australia, \{Rajeev.Gore,Florian.Widmann\}@anu.edu.au
}

\begin{abstract}
We give an optimal (EXPTIME), sound and complete tableaubased algorithm for deciding satisfiability for propositional dynamic logic with converse (CPDL) which does not require the use of analytic cut. Our main contribution is a sound method to combine our previous optimal method for tracking least fix-points in PDL with our previous optimal method for handling converse in the description logic $A L C I$. The extension is non-trivial as the two methods cannot be combined naively. We give sufficient details to enable an implementation by others. Our OCaml implementation seems to be the first theorem prover for CPDL.
\end{abstract}

\section{Introduction}

Propositional dynamic logic (PDL) is an important logic for reasoning about programs. Its formulae consist of traditional Boolean formulae plus "action modalities" built from a finite set of atomic programs using sequential composition (; ), non-deterministic choice $(\cup)$, repetition $(*)$, and test (?). The logic CPDL is obtained by adding converse $\left({ }^{-}\right)$, which allows us to reason about previous actions. The satisfiability problem for CPDL is EXPTIME-complete [1].

De Giacomo and Massacci [2] give an NEXPTIME tableau algorithm for deciding CPDL-satisfiability, and discuss ways to obtain optimality, but do not give an actual EXPTime algorithm. The tableau method of Nguyen and Szałas [3] is optimal. Neither method has been implemented, and since both require an explicit analytic cut rule, it is not at all obvious that they can be implemented efficiently. Optimal game-theoretic methods for fix-point logics [4] can be adapted to handle CPDL [5] but involve significant non-determinism. Optimal automata-based methods [6] for fix-point logics are still in their infancy because good optimisations are not known. We know of no resolution methods for CPDL.

We give an optimal tableau method for deciding CPDL-satisfiability which does not rely on a cut rule. Our main contribution is a sound method to combine our method for tracking and detecting unfulfilled eventualities as early as possible in PDL [7] with our method for handling converse for $A L C I$ [8]. The extension is non-trivial as the two methods cannot be combined naively.

\footnotetext{
* NICTA is funded by the Australian Government's Department of Communications, Information Technology and the Arts and the Australian Research Council through Backing Australia's Ability and the ICT Centre of Excellence program.
} 
Table 1. Smullyan's $\alpha$ - and $\beta$-notation to classify formulae

\begin{tabular}{|c|c|c|c|c|c|c|c|c|c|c|c|}
\hline$\alpha$ & $\varphi \wedge \psi$ & {$[\gamma \cup \delta] \varphi$} & {$[\gamma *] \varphi$} & $\langle\psi ?\rangle \varphi$ & $\langle\gamma ; \delta\rangle \varphi$ & {$[\gamma ; \delta] \varphi$} \\
\hline$\alpha_{1}$ & $\varphi$ & {$[\gamma] \varphi$} & $\varphi$ & $\varphi$ & $\langle\gamma\rangle\langle\delta\rangle \varphi$ & {$[\gamma][\delta] \varphi$} \\
\hline$\alpha_{2}$ & $\psi$ & {$[\delta] \varphi$} & {$[\gamma][\gamma *] \varphi$} & $\psi$ & & & $\varphi \vee \beta_{1}$ & $\varphi$ & $\langle\gamma \cup \delta\rangle \varphi$ & $\langle\gamma *\rangle \varphi$ & {$[\psi ?] \varphi$} \\
\hline$\beta_{2}$ & $\psi$ & $\langle\delta\rangle \varphi$ & $\langle\gamma\rangle\langle\gamma *\rangle \varphi$ & $\sim \psi$ \\
\hline
\end{tabular}

We present a mixture of pseudo code and tableau rules rather than a set of traditional tableau rules to enable easy implementation by others. Our unoptimised OCaml implementation appears to be the first automated theorem prover for CPDL (http://rsise.anu.edu.au/ rpg/CPDLTabProver/). The proofs can be found in the appendix.

\section{Syntactic Preliminaries}

Definition 1. Let $\mathrm{AFml}$ and $\mathrm{APrg}$ be two disjoint and countably infinite sets of propositional variables and atomic programs, respectively. The set LPrg of literal programs is defined as $\operatorname{LPrg}:=\operatorname{APrg} \cup\left\{a^{-} \mid a \in \mathrm{APrg}\right\}$. The set $\mathrm{Fml}$ of all formulae and the set $\operatorname{Prg}$ of all programs are defined mutually inductively as follows where $p \in \mathrm{AFml}$ and $l \in \mathrm{LPrg}$ :

Fml $\quad \varphi::=p|\neg \varphi| \varphi \wedge \varphi|\varphi \vee \varphi|\langle\gamma\rangle \varphi \mid[\gamma] \varphi$

$\operatorname{Prg} \quad \gamma::=l|\gamma ; \gamma| \gamma \cup \gamma|\gamma *| \varphi$ ?.

$A\langle\mathrm{l}\rangle\rangle$-formula is a formula $\langle\gamma\rangle \varphi$ where $\gamma \in \mathrm{LPrg}$ is a $\underline{\text { literal }} \underline{\mathrm{p}}$ rogram.

Implication $(\rightarrow)$ and equivalence $(\leftrightarrow)$ are not part of the core language but can be defined as usual. In the rest of the paper, let $p \in \mathrm{AFml}$ and $l \in \mathrm{LPrg}$.

We omit the semantics as it is a straightforward extension of PDL [7] and write $M, w \Vdash \varphi$ if $\varphi \in \mathrm{Fml}$ holds in the world $w \in W$ of the model $M$.

Definition 2. For a literal program $l \in \mathrm{LPrg}$, we define $l^{\triangleleft}$ as a if $l$ is of the form $a^{-}$, and as $l^{-}$otherwise. A formula $\varphi \in \mathrm{Fml}$ is in negation normal form if the symbol $\neg$ appears only directly before propositional variables. For every $\varphi \in \mathrm{Fml}$, we can obtain a formula $\operatorname{nnf}(\varphi)$ in negation normal form by pushing negations inward such that $\varphi \leftrightarrow \operatorname{nnf} \varphi$ is valid. We define $\sim \varphi:=\operatorname{nnf}(\neg \varphi)$.

We categorise formulae as $\alpha$ - or $\beta$-formulae as shown in Table 1 so that the formulae of the form $\alpha \leftrightarrow \alpha_{1} \wedge \alpha_{2}$ and $\beta \leftrightarrow \beta_{1} \vee \beta_{2}$ are valid. An eventuality is a formula of the form $\left\langle\gamma_{1}\right\rangle \ldots\left\langle\gamma_{k}\right\rangle\langle\gamma *\rangle \varphi$, and Ev is the set of all eventualities. Using Table 1, the binary relation " $\rightsquigarrow$ " relates a \langle\rangle -formulae $\alpha$ (respectively $\beta$ ), to its reduction $\alpha_{1}$ (respectively $\beta_{1}$ and $\beta_{2}$ ). See [7, Def. 7] for their formal definitions.

\section{An Overview of our Algorithm}

Our algorithm builds an and-or graph $G$ by repeatedly applying four rules (see Table 2) to try to build a model for a given $\phi$ in negation normal form. Each node $x$ carries a formula set $\Gamma_{x}$, a status sts $x$, and other fields to be described 
shortly. Rule 1 applies the usual expansion rules to a node to create its children. These expansion rules capture the semantics of CPDL. We use Smullyan's $\alpha / \beta$ rule notation for classifying rules and nodes. As usual, a node $x$ is a ("saturated") state if no $\alpha / \beta$-rule can be applied to it. If $x$ is a state then for each $\langle l\rangle \xi$ in $\Gamma_{x}$, we create a node $y$ with $\Gamma_{y}=\{\xi\} \cup \Delta$, where $\Delta=\left\{\psi \mid[l] \psi \in \Gamma_{x}\right\}$, and add an edge from $x$ to $y$ labelled with $\langle l\rangle \xi$ to record that $y$ is an $l$-successor of $x$.

If $\Gamma_{x}$ contains an obvious contradiction during expansion, its status becomes "closed", which is irrevocable. Else, at some later stage, Rule 2 determines its status as either "closed" or "open". "Open" nodes contain additional information which depends on the status of other nodes. Hence, if a node changes its status, it might affect the status of another ("open") node. If the stored status of a node does not match its current status, the node is no longer up-to-date. Rule 3, which may be applied multiple times to the same node, ensures that "open" nodes are kept up-to-date by recomputing their status if necessary. Finally, Rule 4 detects eventualities which are impossible to fulfil and closes nodes which contain them. We first describe the various important components of our algorithm separately.

Global State Caching. For optimality, the graph $G$ never contains two state nodes which carry the same set of formulae [8]. However, there may be multiple non-states which carry the same set of formulae. That is, a non-state node $x$ carrying $\Gamma$ which appears while saturating a child $y$ of a state $z$ is unique to $y$. If a node carrying $\Gamma$ is required in some other saturation phase, a new node carrying $\Gamma$ is created. Hence the nodes of two saturation phases are distinct.

Converse. Suppose state $y$ is a descendant of an $l$-successor of a state $x$, with no intervening states. Call $x$ the parent state of $y$ since all intervening nodes are not states. We require that $\left\{\psi \mid\left[l^{-}\right] \psi \in \Gamma_{y}\right\} \subseteq \Gamma_{x}$, since $y$ is then compatible with being a $l$-successor of $x$ in the putative model under construction. If some $\left[l^{-}\right] \psi \in$ $\Gamma_{y}$ has $\psi \notin \Gamma_{x}$ then $x$ is "too small", and must be "restarted" as an alternative node $x^{+}$containing all such $\psi$. If any such $\psi$ is a complex formula to which an $\alpha / \beta$-rule is applicable then $x^{+}$is not a state and may have to be "saturated" further. The job of creating these alternatives is done by special nodes [8]. Each special node monitors a state and creates the alternatives when needed.

Detecting Fulfilled and Unfulfilled Eventualities. Suppose the current node $x$ contains an eventuality $e_{x}$. There are three possibilities. The first is that $e_{x}$ can be fulfilled in the part of the graph which is "older" than $x$. Else, it may be possible to reach a node $z$ in the parts of the graph "newer" than $x$ such that $z$ contains a reduction $e_{z}$ of $e_{x}$. Since this "newer" part of the graph is not fully explored yet, future expansions may enable us to fulfil $e_{x}$ via $z$, so the pair $\left(z, e_{z}\right)$ is a "potential rescuer" of $e_{x}$. The only remaining case is that $e_{x}$ cannot be fulfilled in the "older" part of the graph, and has no potential rescuers. Thus future expansions of the graph cannot possibly help to fulfil $e_{x}$ since it cannot reach these "newer" parts of the future graph. In this case $x$ can be "closed". The technical machinery to maintain this information for PDL is from [7]. However, 
the presence of "converse" and the resulting need for alternative nodes requires a more elaborate scheme for CPDL.

\section{The Algorithm}

Our algorithm builds a directed graph $G$ consisting of nodes and directed edges. We first explain the structure of $G$ in more detail.

Definition 3. Let $X$ and $Y$ be sets. We define $X^{\perp}:=X \uplus\{\perp\}$ where $\perp$ indicates the undefined value and $\uplus$ is the disjoint union. If $f: X \rightarrow Y$ is a function and $x \in X$ and $y \in Y$ then the function $f[x \mapsto y]: X \rightarrow Y$ is defined as $f[x \mapsto y]\left(x^{\prime}\right):=y$ if $x^{\prime}=x$ and $f[x \mapsto y]\left(x^{\prime}\right):=f\left(x^{\prime}\right)$ if $x^{\prime} \neq x$.

Definition 4. Let $G=(V, E)$ be a graph where $V$ is a set of nodes and $E$ is a set of directed edges. Each node $x \in V$ has six attributes: $\Gamma_{x} \subseteq \mathrm{Fml}, \operatorname{ann}_{x}$ : $\mathrm{Ev} \rightarrow \mathrm{Fml}^{\perp}$, pst $_{x} \in V^{\perp}, \operatorname{ppr}_{x} \in \mathrm{LPrg}^{\perp}, \mathrm{idx}_{x} \in N a t^{\perp}$, and $\mathrm{sts}_{x} \in \mathfrak{S}$ where $\mathfrak{S}:=$ $\{$ unexp, undef $\} \cup\{$ closed(alt) $\mid$ alt $\subseteq \mathscr{P}(\mathrm{Fml})\} \cup\{$ open(prs, alt $) \mid$ prs $: \mathrm{Ev} \rightarrow$ $(\mathscr{P}(V \times \mathrm{Ev}))^{\perp} \&$ alt $\left.\subseteq \mathscr{P}(\mathrm{Fml})\right\}$. Each directed edge $e \in E$ is labelled with a label $l_{e} \in(\mathrm{Fml} \cup \mathscr{P}(\mathrm{Fml}) \cup\{\mathrm{cs}\})^{\perp}$ where cs is just a constant.

All attributes of a node $x \in V$ are initially set at the creation of $x$, possibly with the value $\perp$ (if allowed). Only the attributes $\operatorname{idx}_{x}$ and $\operatorname{sts}_{x}$ are changed at a later time. We use the function create-new-node $(\Gamma$, ann, pst, ppr, idx, sts) to create a new node and initialise its attributes in the obvious way.

The finite set $\Gamma_{x}$ contains the formulae which are assigned to $x$. The attribute $\operatorname{ann}_{x}$ is defined for the eventualities in $\Gamma_{x}$ at most. If $\operatorname{ann}_{x}(\varphi)=\varphi^{\prime}$ then $\varphi^{\prime} \in \Gamma_{x}$ and $\varphi \rightsquigarrow \varphi^{\prime}$. The intuitive meaning is that $\varphi$ has already been "reduced" to $\varphi^{\prime}$ in $x$. For a state (as defined below) we always have that $\operatorname{ann}_{x}$ is undefined everywhere since we do not need the attribute for states.

The node $x$ is called a state iff both attributes $\mathrm{pst}_{x}$ and $\mathrm{ppr}_{x}$ are undefined. For all other nodes, the attribute $\mathrm{pst}_{x}$ identifies the, as we will ensure, unique ancestor $p \in V$ of $x$ such that $p$ is a state and there is no other state between $p$ and $x$ in $G$. We call $p$ the parent state of $x$. The creation of the child of $p$ which lies on the path from $p$ to $x$ (it could be $x$ ) was caused by a $\langle\mathrm{lp}\rangle$-formula $\langle l\rangle \varphi$ in $\Gamma_{p}$. The literal program $l$ which we call the parent program of $x$ is stored in $\operatorname{ppr}_{x}$. Hence, for nodes which are not states, both pst ${ }_{x}$ and $\operatorname{ppr}_{x}$ are defined.

The attribute $\operatorname{sts}_{x}$ describes the status of $x$. Unlike the attributes described so far, its value may be modified several times. The value unexp, which is the initial value of each node, indicates that the node has not yet been expanded. When a node is expanded, its status becomes either closed( $(\cdot)$ if it contains an immediate contradiction, or undef to indicate that the node has been expanded but that its "real" status is to be determined. Eventually, the status of each node is set to either $\operatorname{closed}(\cdot)$ or open $(\cdot, \cdot)$. If the status is open $(\cdot, \cdot)$, it might be modified several times later on, either to $\operatorname{closed}(\cdot)$ or to open $(\cdot, \cdot)$ (with different arguments), but once it becomes closed(·), it will never change again.

We call a node undefined if its status is unexp or undef and defined otherwise. Hence a node is undefined initially, becomes defined eventually, and 
then never becomes undefined again. Furthermore, we call $x$ closed iff its status is closed(alt) for some alt $\subseteq \mathscr{P}(\mathrm{Fml})$. In this case, we define alt $x:=$ alt. We call $x$ open iff its status is open(prs, alt) for some prs : $\mathrm{Ev} \rightarrow(\mathscr{P}(V \times \mathrm{Ev}))^{\perp}$ and some alt $\subseteq \mathscr{P}(\mathrm{Fml})$. In this case, we define $\operatorname{prs}_{x}:=$ prs and alt $x:=$ alt. To avoid some clumsy case distinctions, we define alt $x:=\emptyset$ if $x$ is undefined.

The value closed(alt) indicates that the node is "useless" for building an interpretation because it is either unsatisfiable or "too small". In the latter case, the set alt of alternative sets contains information about missing formulae. Finally, the value open(prs, alt) indicates that there is still hope that $x$ is "useful" and the function $\operatorname{prs}_{x}$ contains information about each eventuality $e_{x} \in \Gamma_{x}$ as explained in the overview. Although $x$ itself may be useful, we need its alternative sets in case it becomes closed later on. Hence it also has a set of alternative sets.

The attribute $\mathrm{idx}_{x}$ serves as a time stamp. It is set to $\perp$ at creation time of $x$ and becomes defined when $x$ becomes defined. When this happens, the value of $\mathrm{idx}_{x}$ is set such that $\mathrm{idx}_{x}>\mathrm{idx}_{y}$ for all nodes $y$ which became defined earlier than $x$. We define $y \sqsubset x$ iff $\operatorname{idx}_{y} \neq \perp$ and either $\operatorname{idx}_{x}=\perp$ or $\operatorname{idx}_{y}<\operatorname{idx}_{x}$. Note that $y \sqsubset x$ depends on the current state of the graph. However, once $y \sqsubset x$ holds, it will do so for the rest of the time.

To track eventualities, we label an edge between a state and one of its children by the $\langle\mathrm{lp}\rangle$-formula $\langle l\rangle \varphi$ which creates this child. Additionally, we label edges from special nodes (see overview) to their corresponding states with the marker cs. We also label edges from special nodes to its alternative nodes with the corresponding alternative set.

Definition 5. Let $\mathrm{ann}^{\perp}: \mathrm{Ev} \rightarrow \mathrm{Fml}^{\perp}$ and $\mathrm{prs}^{\perp}: \mathrm{Ev} \rightarrow(\mathscr{P}(V \times \mathrm{Ev}))^{\perp}$ be the functions which are undefined everywhere. For a node $x \in V$ and a label $l \in$ $\mathrm{Fml} \cup \mathscr{P}(\mathrm{Fml}) \cup\{\mathrm{cs}\}$, let getChild $(x, l)$ be the node $y \in V$ such that there exists an edge $e \in E$ from $x$ to $y$ with $l_{e}=l$. If $y$ does not exists or is not unique, let the result be $\perp$. For a function prs $: \mathrm{Ev} \rightarrow(\mathscr{P}(V \times \mathrm{Ev}))^{\perp}$, a node $x \in V$, and an eventuality $\varphi \in \mathrm{Ev}$, we define the set reach $(\mathrm{prs}, x, \varphi)$ of eventualities as follows:

$$
\begin{aligned}
& \operatorname{reach}(\operatorname{prs}, x, \varphi):=\left\{\psi \in \operatorname{Ev} \mid \exists k \in \mathbb{N}_{0} \cdot \exists \varphi_{0}, \ldots, \varphi_{k} \in \operatorname{Ev} .\left(\psi=\varphi_{k} \&\right.\right. \\
&\left.\left.\left(x, \varphi_{0}\right) \in \operatorname{prs}(\varphi) \& \forall i \in\{0, \ldots, k-1\} .\left(x, \varphi_{i+1}\right) \in \operatorname{prs}\left(\varphi_{i}\right)\right)\right\}
\end{aligned}
$$

The function defer : $V \times \mathrm{Ev} \rightarrow \mathrm{Fml}^{\perp}$ is defined as follows:

$$
\operatorname{defer}(x, \varphi):=\left\{\begin{aligned}
\psi & \text { if } \exists k \in \mathbb{N}_{0} \cdot \exists \varphi_{0}, \ldots, \varphi_{k} \in \mathrm{Fml} .\left(\varphi_{0}=\varphi \& \varphi_{k}=\psi \&\right. \\
& \forall i \in\{0, \ldots, k-1\} .\left(\varphi_{i} \in \operatorname{Ev} \& \operatorname{ann}_{x}\left(\varphi_{i}\right)=\varphi_{i+1}\right) \& \\
& \left.\left(\varphi_{k} \notin \operatorname{Ev} \text { or } \operatorname{ann}_{x}\left(\varphi_{k}\right)=\perp\right)\right) \\
\perp \text { otherwise. } &
\end{aligned}\right.
$$

The function getChild $(x, l)$ retrieves a particular child of $x$. It is easy to see that, during the algorithm, the child is always unique if it exists.

Intuitively, the function reach(prs, $x, \varphi)$ computes all eventualities which can be "reached" from $\varphi$ inside $x$ according to prs. If a potential rescuer $(x, \psi)$ is 


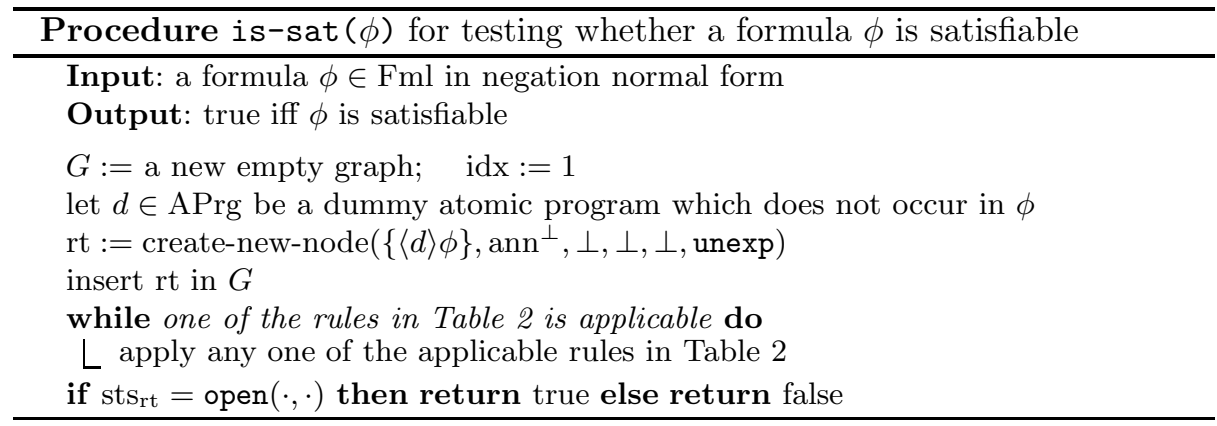

Table 2. Rules used in the procedure is-sat

\begin{tabular}{|c|c|}
\hline $\begin{array}{l}\text { Rule 1: } \\
\text { Condition: } \\
\text { Action: }\end{array}$ & $\begin{array}{l}\text { Some node } x \text { has not been expanded yet. } \\
\exists x \in V \cdot \text { sts }_{x}=\text { unexp } \\
\text { expand }(x)\end{array}$ \\
\hline $\begin{array}{l}\text { Rule 2: } \\
\text { Condition: } \\
\text { Action: }\end{array}$ & $\begin{array}{l}\text { The status of some node } x \text { is still undefined. } \\
\exists x \in V \cdot \operatorname{sts}_{x}=\text { undef } \\
\operatorname{sts}_{x}:=\operatorname{det}-\operatorname{status}(x) \quad \& \operatorname{idx}_{x}:=\mathrm{idx} \quad \& \mathrm{idx}:=\mathrm{idx}+1\end{array}$ \\
\hline $\begin{array}{l}\text { Rule 3: } \\
\text { Condition: } \\
\text { Action: }\end{array}$ & $\begin{array}{l}\text { Some open node } x \text { is not up-to-date. } \\
\exists x \in V \text {. open }(\cdot, \cdot)=\operatorname{sts}_{x} \neq \operatorname{det}-\operatorname{status}(x) \\
\operatorname{sts}_{x}:=\operatorname{det}-\operatorname{status}(x)\end{array}$ \\
\hline $\begin{array}{l}\text { Rule 4: } \\
\text { Condition: } \\
\text { Action: }\end{array}$ & 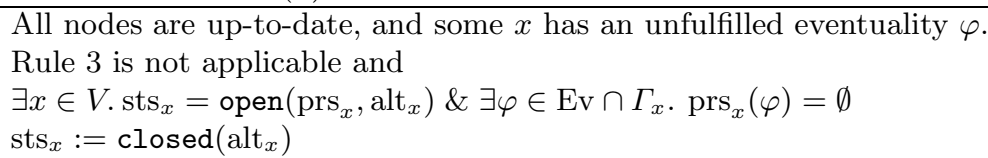 \\
\hline
\end{tabular}

contained in $\operatorname{prs}(\varphi)$, the potential rescuers of $\psi$ are somehow relevant for $\varphi$ at $x$. Therefore $\psi$ itself is relevant for $\varphi$ at $x$. The function $\operatorname{reach}(\operatorname{prs}, x, \varphi)$ computes exactly the transitive closure of this relevance relation.

Intuitively, the function $\operatorname{defer}(x, \varphi)$ follows the "ann $x_{x}$-chain". That is, it computes $\varphi_{1}:=\operatorname{ann}_{x}(\varphi), \varphi_{2}:=\operatorname{ann}_{x}\left(\varphi_{1}\right)$, and so on. There are two possible outcomes. The first outcome is that we eventually encounter a $\varphi_{k}$ which is either not an eventuality or has $\operatorname{ann}_{x}\left(\varphi_{k}\right)=\perp$. Consequently, we cannot follow the "ann $x^{-}$ chain" any more. In this case we stop and return defer $(x, \varphi):=\varphi_{k}$. The second outcome is that we can follow the "ann $x_{x}$-chain" indefinitely. Then, as $\Gamma_{x}$ is finite, there must exist a cycle $\varphi_{0}, \ldots, \varphi_{n}, \varphi_{0}$ of eventualities such that $\operatorname{ann}_{x}\left(\varphi_{i}\right)=\varphi_{i+1}$ for all $0 \leq i<n$, and $\operatorname{ann}_{x}\left(\varphi_{n}\right)=\varphi_{0}$. In this case we say that $x$ (or $\Gamma_{x}$ ) contains an "at a world" cycle and return $\operatorname{defer}(x, \varphi):=\perp$.

Next we comment on all procedures given in pseudocode.

Procedure is-sat $(\phi)$ is invoked to determine whether a formula $\phi \in \mathrm{Fml}$ in negation normal form is satisfiable. It creates a root node $\mathrm{rt}$ and initialises the graph $G$ to contain only rt. The dummy program $d$ is used to make rt a state so that each node in $G$ which is not a state has a parent state. The global variable idx is used to set the time stamps of the nodes accordingly. 
While at least one of the rules in Table 2 is applicable, that is its condition is true, the algorithm applies any applicable rule. If no rules are applicable, the algorithm returns satisfiable iff $\mathrm{rt}$ is open.

Rule 1 picks an unexpanded node and expands it. Rule 2 picks an expanded but undefined node and computes its (initial) status. It also sets the correct time stamp. Rule 3 picks an open node whose status has changed and recomputes its status. Its meaning is, that if we compute $\operatorname{det}-\operatorname{status}(x)$ on the current graph then its result is different from the value in sts $_{x}$, and consequently, we update $\operatorname{sts}_{x}$ accordingly. Rule 4 is only applicable if all nodes are up-to-date. It picks an open node containing an eventuality $\varphi$ which is currently not fulfilled in the graph and which does not have any potential rescuers either. As this indicates that $\varphi$ can never be fulfilled, the node is closed.

This description leaves several questions open, most notably: "How do we check efficiently whether Rule 3 is applicable?" and "Which rule should be taken if several rules are applicable?". We address these issues in Section 5.

Procedure expand $(x)$ expands a node $x$. If $\Gamma_{x}$ contains an immediate contradiction or an "at a world" cycle then we close $x$ and set the time stamp accordingly. For the other cases, we assume implicitly that $\Gamma_{x}$ does not contain either of these.

If $x$ is a state, that is $\mathrm{pst}_{x}=\perp$, then we do the following for each $\langle\mathrm{lp}\rangle$ formula $\left\langle l_{i}\right\rangle \varphi_{i}$. We create a new node $y_{i}$ whose associated set contains $\varphi_{i}$ and all $\psi$ such that $\left[l_{i}\right] \psi \in \Gamma_{x}$. As none of the eventualities in $\Gamma_{y_{i}}$ is reduced yet, there are no annotations. The parent state of $y_{i}$ is obviously $x$ and its parent program is $l_{i}$. In order to relate $y_{i}$ to $\left\langle l_{i}\right\rangle \varphi_{i}$, we label the edge from $x$ to $y_{i}$ with $\left\langle l_{i}\right\rangle \varphi_{i}$. We call $y_{i}$ the successor of $\left\langle l_{i}\right\rangle \varphi_{i}$.

If $x$ is not a state and $\Gamma_{x}$ contains an $\alpha$-formula $\alpha$ whose decompositions are not in $\Gamma_{x}$, or which is an unannotated eventuality, we call $x$ an $\alpha$-node. In this case, we create a new node $y$ whose associated set is the result of adding all decompositions of $\alpha$ to $\Gamma_{x}$. If $\alpha$ is an eventuality then $\operatorname{ann}_{y}$ extends $a_{n}$ by mapping $\alpha$ to $\alpha_{1}$. The parent state and the parent program of $y$ are inherited from $x$. Note that $\operatorname{pst}_{x}$ and $\operatorname{ppr}_{x}$ are defined as $x$ is not a state. Also note that $\Gamma_{y} \supsetneq \Gamma_{x}$ or $\alpha$ is an eventuality which is annotated in $\operatorname{ann}_{y}$ but not in $\operatorname{ann}_{x}$.

If $x$ is neither a state nor an $\alpha$-node and $\Gamma_{x}$ contains a $\beta$-formula $\beta$ such that neither of its immediate subformulae is in $\Gamma_{x}$, or such that $\beta$ is an unannotated eventuality, we call $x$ a $\beta$-node. For each decomposition $\beta_{i}$ we do the following. We create a new node $y_{i}$ whose associated set is the result of adding $\beta_{i}$ to $\Gamma_{x}$. If $\beta$ is an eventuality then $\operatorname{ann}_{y_{i}}$ extends $\operatorname{ann}_{x}$ by mapping $\alpha$ to $\beta_{i}$. The parent state and the parent program of $y$ are inherited from $x$. Note that pst ${ }_{x}$ and $\operatorname{ppr}_{x}$ are defined as $x$ is not a state. Also note that $\Gamma_{y_{i}} \supsetneq \Gamma_{x}$ or $\beta$ is an eventuality which is annotated in $\operatorname{ann}_{y_{i}}$ but not in $\operatorname{ann}_{x}$.

If $x$ is neither a state nor an $\alpha$-node nor a $\beta$-node, it must be fully saturated and we call it a special node. Intuitively, a special node sits between a saturation phase and a state and is needed to handle the "special" issue arising from converse programs, as explained in the overview. Like $\alpha$ - and $\beta$-nodes, special nodes have a unique parent state and a unique parent program. In this case we check whether there already exists a state $y$ in $G$ which has the same set of formulae 


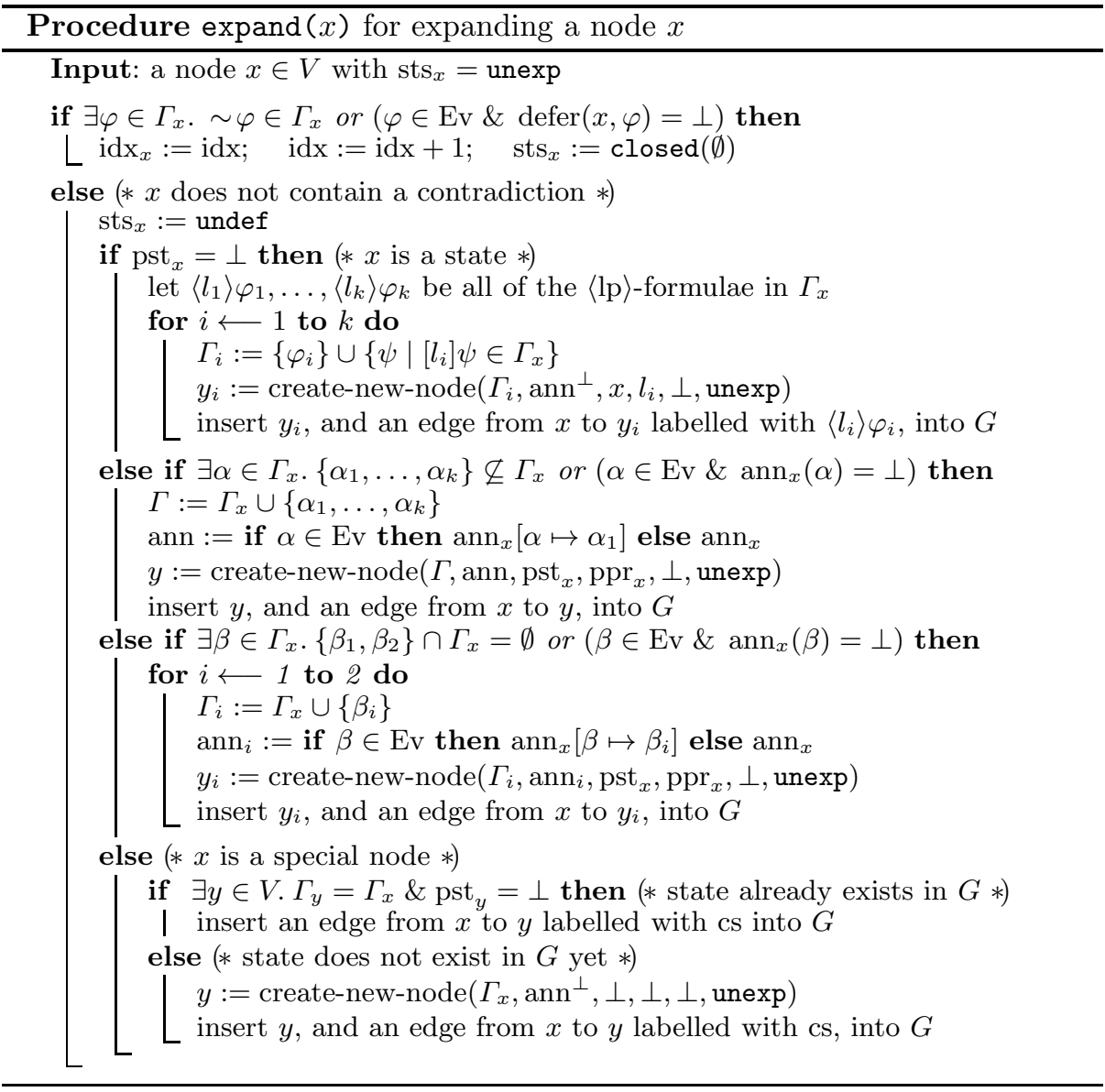

as the special node. If such a state $y$ exists, we link $x$ to $y$; else we create such a state and link $x$ to it. In both cases we label the edge with the marker cs since a special node can have several children (see below) and we want to uniquely identify the cs-child $y$ of $x$. Note that there is only at most one state for each set of formulae and that states are always fully saturated since special nodes are.

Procedure det-status $(x)$ determines the current status of a node $x$. Its result will always be $\operatorname{closed}(\cdot)$ or open $(\cdot, \cdot)$. If $x$ is an $\alpha / \beta$-node or a state, the procedure just calls the corresponding sub-procedure. If $x$ is a special node, we determine the set $\Gamma_{\text {alt }}$ of all formulae $\varphi$ such that $\left[\operatorname{ppr}_{x}^{\smile}\right] \varphi$ is in $\Gamma_{x}$ but $\varphi$ is not in the set of the parent state of $x$. If there is no such formula, that is $\Gamma_{\text {alt }}$ is the empty set, we say that $x$ is compatible with its parent state pst $_{x}$. Note that incompatibilities can only arise because of converse programs.

If $x$ is compatible with pst ${ }_{x}$, all is well, so we determine its status via the corresponding sub-procedure. Else we cannot connect $\mathrm{pst}_{x}$ to a state with $\Gamma_{x}$ assigned to it in the putative model as explained in the overview, and, thus, we 

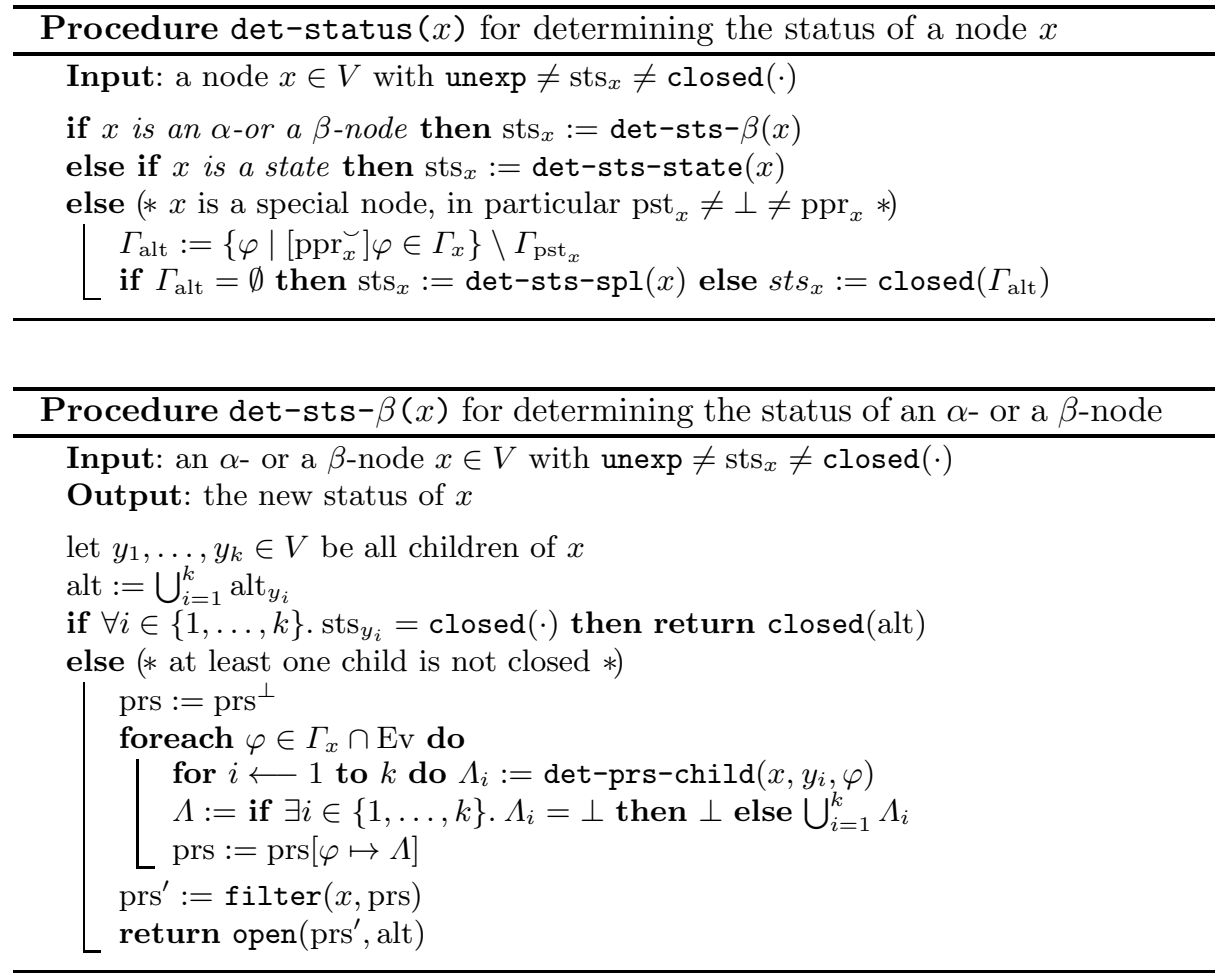

can close $x$. That does not, however, mean that pst $_{x}$ is unsatisfiable; maybe it is just missing some formulae. We cannot extend pst $_{x}$ directly as this may have side-effects elsewhere; but to tell pst ${ }_{x}$ what went wrong, we remember $\Gamma_{\text {alt }}$. The meaning is that if we create an alternative node for pst $_{x}$ by adding the formulae in $\Gamma_{\text {alt }}$, we might be more successful in building an interpretation.

Procedure det-sts- $\beta(x)$ computes the status of an $\alpha$ - or a $\beta$-node $x \in V$. For this task, an $\alpha$-node can be seen as a $\beta$-node with exactly one child. The set of alternative sets of $x$ is the union of the sets of alternative sets of all children. If all children of $x$ are closed then $x$ must also be closed. Otherwise we compute the set of potential rescuers for each eventuality $\varphi$ in $\Gamma_{x}$ as follows. For each child $y_{i}$ of $x$ we determine the potential rescuers of $\varphi$ which result from following $y_{i}$ by invoking det-prs-child. If the set of potential rescuers corresponding to some $y_{i}$ is $\perp$ then $\varphi$ can currently be fulfilled via $y_{i}$ and $\operatorname{prs}_{x}(\varphi)$ is set to $\perp$. Else $\varphi$ cannot currently be fulfilled in $G$, but each child returned a set of potential rescuers, and the set of potential rescuers for $\varphi$ is their union. Finally, we deal with potential rescuers in prs of the form $(x, \chi)$ for some $\chi \in \mathrm{Ev}$ by calling filter.

Procedure det-sts-state $(x)$ computes the status of a state $x \in V$. We obtain the successors for all $\langle\mathrm{lp}\rangle$-formulae in $\Gamma_{x}$. If any successor is closed then $x$ is closed with the same set of alternative sets. Else the set of alternative sets of $x$ is the union of the sets of alternative sets of all children and we compute the potential 


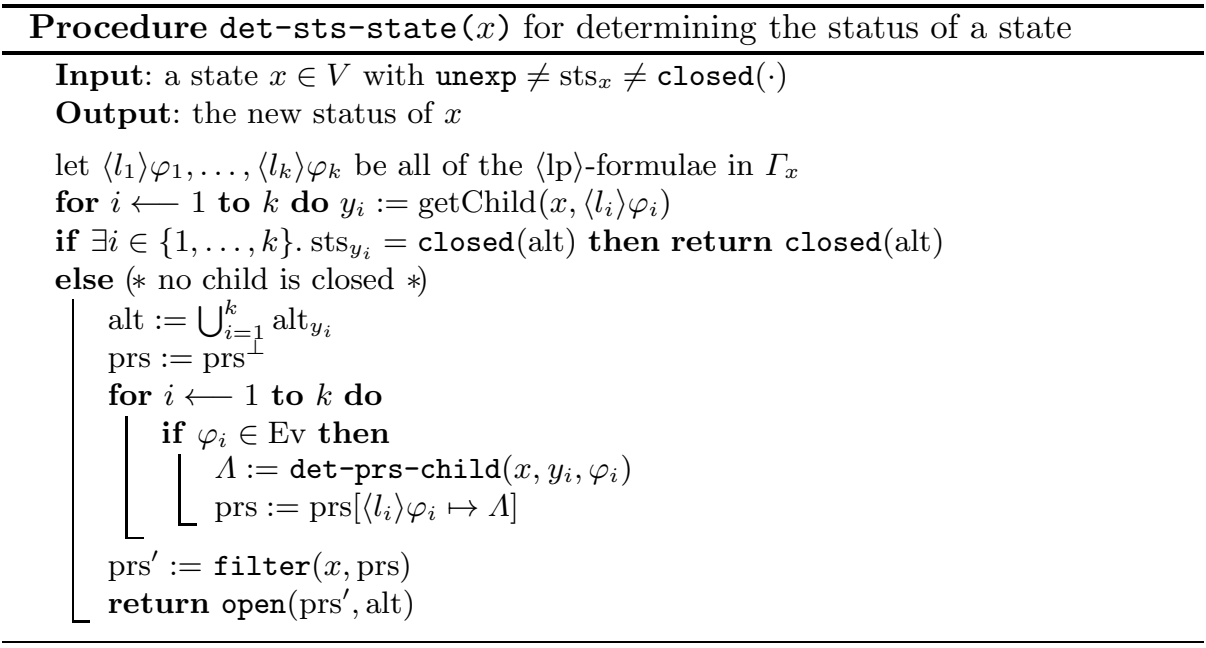

rescuers for each eventuality $\left\langle l_{i}\right\rangle \varphi_{i}$ in $\Gamma_{x}$ by invoking det-prs-child. Finally, we deal with potential rescuers in prs of the form $(x, \chi)$ for some $\chi \in \operatorname{Ev}$ by calling filter. Note that we do not consider eventualities which are not $\langle\mathrm{lp}\rangle$ formulae. The intuitive reason is that the potential rescuers of such eventualities are determined by following the annotation chain (see below). However, different special nodes which have the same set, and hence all link to $x$, might have different annotations. Hence we cannot (and do not need to) fix the potential rescuer sets for eventualities in $x$ which are not $\langle\mathrm{lp}\rangle$-formulae.

Procedure det-sts-spl $(x)$ computes the status of a special node $x \in V$. First, we retrieve the state $y_{0}$ corresponding to $x$, namely the unique cs-child of $x$. For all alternative sets $\Gamma_{i}$ of $y_{0}$ we do the following. If there does not exist a child of $x$ such that the corresponding edge is labelled with $\Gamma_{i}$, we create a new node $y_{i}$ whose associated set is the result of adding the formulae in $\Gamma_{i}$ to $\Gamma_{x}$. The annotations, the parent state, and the parent program of $y_{i}$ are inherited from $x$. We label the new edge from $x$ to $y_{i}$ with $\Gamma_{i}$. In other words we unpack the information stored in the alternative sets in alt $y_{0}$ into actual nodes which are all children of $x$. Note that each $\Gamma_{i} \neq \emptyset$ by construction in det-status. Some children of $x$ may not be referenced from alt $y_{0}$, but we consider them anyway.

The set of alternative sets of $x$ is the union of the sets of alternative sets of all children; with the exception of $y_{0}$ since the alternative sets of $y_{0}$ are not related to pst $_{x}$ but affect $x$ directly as we have seen. If all children of $x$ are closed then $x$ must also be closed. Otherwise we compute the set of potential rescuers for each eventuality $\varphi$ in $\Gamma_{x}$ as follows.

First, we determine $\varphi^{\prime}:=\operatorname{defer}(x, \varphi)$. Note that $\varphi^{\prime}$ is defined because the special node $x$ cannot contain an "at a world" cycle by definition. If $\varphi^{\prime}$ is not an eventuality then $\varphi^{\prime}$ is fulfilled in $x$ and $\operatorname{prs}(\varphi)$ remains $\perp$. If $\varphi^{\prime}$ is an eventuality, it must be a $\langle$ lp $\rangle$-formula as $x$ is a special node. We use $\varphi^{\prime}$ instead of $\varphi$ since only $\langle\mathrm{l}\rangle\rangle$-formula have a meaningful interpretation in $\operatorname{prs}_{y_{0}}$ (see above). 


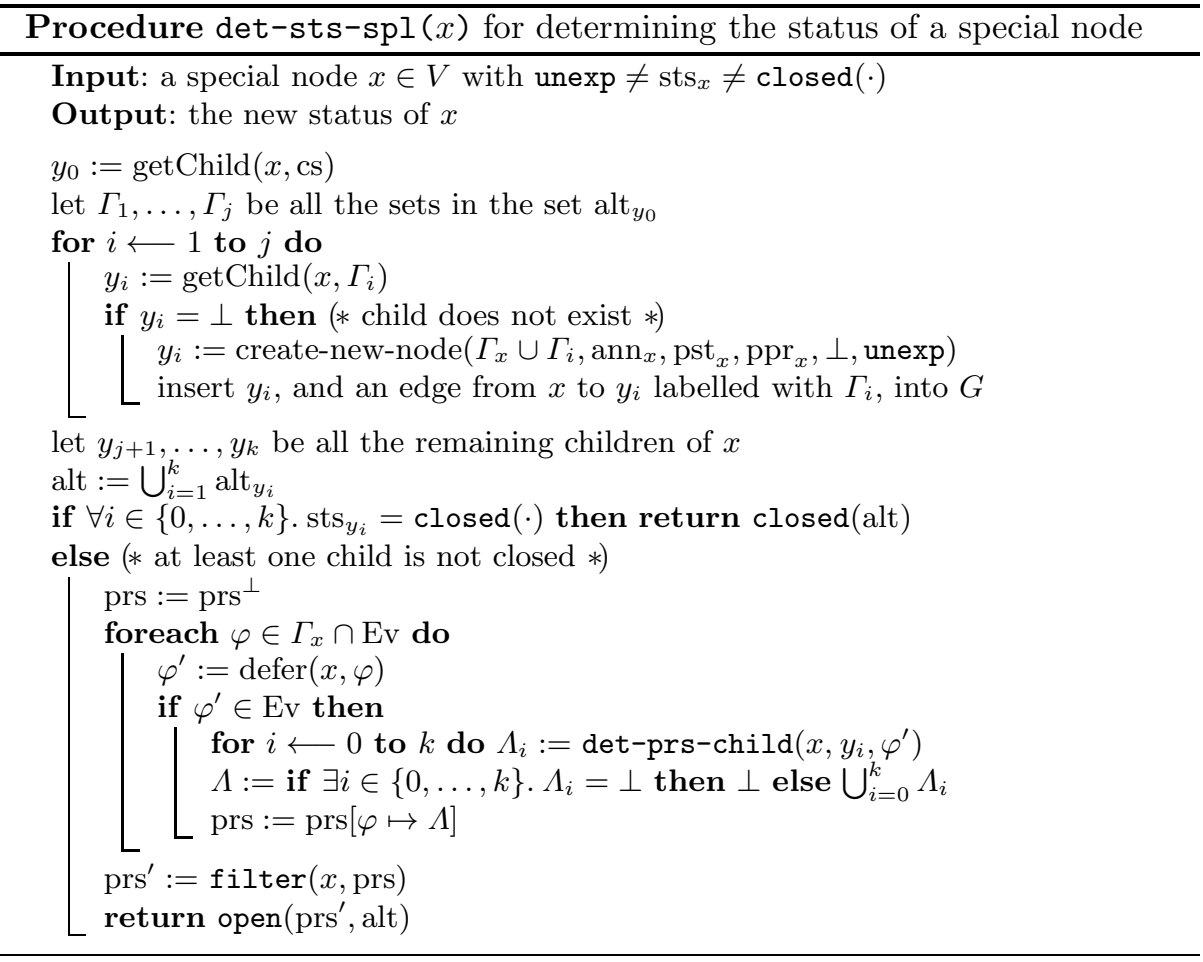

For each child $y_{i}$ of $x$ we determine the potential rescuers of $\varphi^{\prime}$ by invoking det-prs-child. If the set of potential rescuers corresponding to some $y_{i}$ is $\perp$

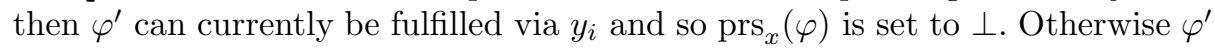
cannot currently be fulfilled in $G$, but each child returned a set of potential rescuers, and the set of potential rescuers for $\varphi$ is their union. Finally, we deal with potential rescuers in prs of the form $(x, \chi)$ for some $\chi \in$ Ev by calling filter.

Procedure det-prs-child $(x, y, \varphi)$ determines whether an eventuality $\psi \in \Gamma_{x}$, which is not passed as an argument, can be fulfilled via $y$ such that $\varphi$ is part of the corresponding fulfilling path; or else which potential rescuers $\psi$ can reach via $y$ and $\varphi$. If $y$ is closed, it cannot help to fulfil $\psi$ as indicated by the empty set. If $x$ is undefined or did not become defined before $x$ then $(y, \varphi)$ itself is a potential rescuer of $x$. Else, if $\varphi$ can be fulfilled, i.e. $\operatorname{prs}_{y}(\varphi)=\perp$, then $\psi$ can be fulfilled too, so we return $\perp$. Otherwise we invoke the procedure recursively on all potential rescuers in $\operatorname{prs}_{y}(\varphi)$. If at least one of these invocations returns $\perp$ then $\psi$ can be fulfilled via $y$ and $\varphi$ and the corresponding rescuer in $\operatorname{prs}_{y}(\varphi)$. If all invocations return a set of potential rescuers, the set of potential rescuers for $\psi$ is their union. The recursion is well-defined because if $\left(z_{i}, \varphi_{i}\right) \in \operatorname{prs}_{y}(\varphi)$ then either $z_{i}$ is still undefined or $z_{i}$ became defined later than $y$.

Each invocation of det-prs-child can be uniquely assigned to the invocation of det-sts- $\beta$, det-sts-state, or det-sts-spl which (possibly indi- 
rectly) invoked it. To meet our complexity bound, we require that under the same invocation of det-sts- $\beta$, det-sts-state, or det-sts-spl, the procedure det-prs-child is only executed at most once for each argument triple. Instead of executing it a second time with the same arguments, it uses the cached result of the first invocation. Since det-prs-child does not modify the graph, the second invocation would return the same result as the first one. An easy implementation of the cache is to store the result of det-prs-child $(x, y, \varphi)$ in the node $y$ together with $\varphi$ and a unique id number for each invocation of det-sts- $\beta$, det-sts-state, or det-sts-spl.

Procedure filter $(x$, prs $)$ deals with the potential rescuers for each eventuality of a node $x$ which are of the form $(x, \psi)$ for some $\psi \in \mathrm{Ev}$. The second argument of filter is a provisional prs for $x$. If an eventuality $\varphi \in \Gamma_{x}$ is currently fulfillable in $G$ there is nothing to be done, so let $(x, \psi) \in \operatorname{prs}(\varphi)$. If $\psi=\varphi$ then $(x, \varphi)$ cannot be a potential rescuer for $\varphi$ in $x$ and should not appear in $\operatorname{prs}(\varphi)$. But what about potential rescuers of the form $(x, \psi)$ with $\psi \neq \varphi$ ? Since we want the nodes in the potential rescuers to become defined later than $x$, we cannot keep $(x, \psi)$ in $\operatorname{prs}(\varphi)$; but we cannot just ignore the pair either.

Intuitively $(x, \psi) \in \operatorname{prs}(\varphi)$ means that $\varphi \in \Gamma_{x}$ can "reach" $\psi \in \Gamma_{x}$ by following a loop in $G$ which starts at $x$ and returns to $x$ itself. Thus if $\psi$ can be fulfilled in $G$, so can $\varphi$; and all potential rescuers of $\psi$ are also potential rescuers of $\varphi$. The function reach(prs, $x, \varphi)$ computes all eventualities in $x$ which are "reachable" from $\varphi$ in the sense above, where transitivity is taken into account. That is, it detects all self-loops from $x$ to itself which are relevant for fulfilling $\varphi$. We add $\varphi$ as it is not in reach(prs, $x, \varphi$ ). If any of these eventualities is fulfilled in $G$ then $\varphi$ can be fulfilled and is consequently undefined in the resulting prs'. Otherwise we take all their potential rescuers whose nodes are not $x$.

Theorem 6 (Soundness, Completeness and Complexity). Let $\phi \in \mathrm{Fml}$ be a formula in negation normal form of size $n$. The procedure is-sat $(\phi)$ terminates, runs in EXPTIME in $n$, and $\phi$ is satisfiable iff is-sat $(\phi)$ returns true.

\section{Implementation, Optimisations, and Strategy}

It should be fairly straightforward to implement our algorithm. It remains to show an efficient way to find nodes which are not up-to-date. It is not too hard to see that the status of a node $x$ can become outdated only if its children change their status or det-prs-child $(x, y, \cdot)$ was invoked when $x$ 's previous status was determined and $y$ now changes its status. If we keep track of nodes of the second kind by inserting additional "update"-edges as described in [7], we can use a queue for all nodes that might need updating. When the status of a node is modified, we queue all parents and all nodes linked by "update"-edges.

We have omitted several refinements from our description for clarity. The most important is that if a state $s$ is closed, all non-states which have $s$ as a parent state are ignorable since their status cannot influence any other node $t$ unless $t$ also has $s$ as a parent state. Moreover, if every special node parent $x$ of a 
state $s^{\prime}$ is incompatible or itself has a closed parent state, then $s^{\prime}$ and the nodes having $s^{\prime}$ as parent state are ignorable. This applies transitively, but if $s^{\prime}$ gets a new parent whose parent state is not closed then $s^{\prime}$ becomes "active" again.

Another issue is which rule to choose if several are applicable. As we have seen, it is advantageous to close nodes as early as possible. Apart from immediate contradictions, we have Rule 4 which closes a node because it contains an unfulfillable eventuality. If we can apply Rule 4 early while the graph is still small, we might prevent big parts of the graph being built needlessly later. Trying to apply Rule 4 has several consequences on the strategy of how to apply rules.

First, it is important to keep all nodes up-to-date since Rule 4 is not applicable otherwise. Second, it is preferable that a node $x$ cannot reach open nodes which became defined (or will be defined) after $x$ did. Hence, we should try to use Rule 2 on a node only if all children are already defined.

\section{An Example}

To demonstrate how the algorithm works, we invoke it on the satisfiable toy formula $\langle a\rangle \phi$ where $\phi:=\langle a *\rangle\left[a^{-}\right] p$. To save space, Fig. 1 only shows the core subgraph of the tableau. Remember that the order of rule applications is not fixed but the example will follow some of the guidelines given in Section 5 .

The nodes in Fig. 1 are numbered in order of creation. The annotation ann is given using " $\rightsquigarrow$ " in $\Gamma$. For example, in node (3), we have $\Gamma_{3}=\left\{\phi,\left[a^{-}\right] p\right\}$, and $a_{3} n_{3}$ maps the eventuality $\phi$ to $\left[a^{-}\right] p$ and is undefined elsewhere. The bottom line of a node contains the parent state and the parent program on the left, and the time stamp on the right. We do not show the status of a node since it changes during the algorithm, but explain it in the text. If we write $\operatorname{sts}_{x}=\operatorname{open}(\Lambda, \cdot)$ where $\Lambda \subseteq V \times \mathrm{Ev}$, we mean that $\operatorname{prs}_{x}$ maps all eventualities in $\Gamma_{x}$, with the exception of non-〈lp $\rangle$-formulae if $x$ is a state, to $\Lambda$ and is undefined elsewhere.

We only consider the core subgraph of $\phi$ and start by expanding node (1) which creates (2). Then we expand (2) and create (3) and (4) which are both special nodes. Next we expand (3) and create the state (5). Expanding (5) creates no new nodes since $\Gamma_{5}$ contains no $\langle\mathrm{lp}\rangle$-formula. Now we define (5) and then (3). This results in setting $\operatorname{sts}_{5}:=o$ pen $\left(\operatorname{prs}^{\perp}, \emptyset\right)$ according to det-sts-state, and sts $_{3}:=$ closed $(\{p\})$ since (3) is not compatible with its parent state (1). Expanding (4) inserts the edge from (4) to (1) and defining (4) sets sts $4:=\operatorname{open}(\{(1,\langle a\rangle \phi)\}, \emptyset)$ according to det-sts-spl. Note that (6) does not exist yet. Next we define (2) and then (1) which results in setting $\operatorname{sts}_{2}:=\operatorname{open}(\{(1,\langle a\rangle \phi)\},\{p\})$ according to $\operatorname{det}-\mathrm{sts}-\beta$ and $\mathrm{sts}_{1}:=\operatorname{open}(\emptyset,\{p\})$ thanks to filter.

Note that $\langle a\rangle \phi \in \Gamma_{1}$ has an empty set of potential rescuers. In PDL, we could thus close (1), but converse programs complicate matters for CPDL as reflected by the fact that Rule 4 is not applicable for (1) because (4) is not up-to-date. Updating (4) creates (6) and sets $\operatorname{sts}_{4}:=\operatorname{open}(\{(1,\langle a\rangle \phi),(6,\langle a\rangle \phi)\}, \emptyset)$. Updating (2) and then (1) sets $\operatorname{sts}_{2}:=\operatorname{open}(\{(1,\langle a\rangle \phi),(6,\langle a\rangle \phi)\},\{p\})$ and $\operatorname{sts}_{1}:=$ open $(\{(6,\langle a\rangle \phi)\},\{p\})$. Now all nodes are up-to-date, but Rule 4 is not applicable for (1) because the set of potential rescuers for $\phi$ is no longer empty. 
Procedure det-prs-child $(x, y, \varphi)$ for passing a prs-entry of a child to a parent

Input: two nodes $x, y \in V$ and a formula $\varphi \in \Gamma_{y} \cap \mathrm{Ev}$

Output: $\perp$ or a set of node-formula pairs

Remark: if det-prs-child $(x, y, \varphi)$ has been invoked before with exactly the same arguments and under the same invocation of det-sts- $\beta$, det-sts-state or det-sts-spl, the procedure is not executed a second time but returns the cached result of the first invocation. We do not model this behaviour explicitly in the pseudocode.

if $\operatorname{sts}_{y}=\operatorname{closed}(\cdot)$ then return $\emptyset$

else if $\operatorname{sts}_{y}=$ unexp or $\operatorname{sts}_{y}=$ undef or not $y \sqsubset x$ then return $\{(y, \varphi)\}$

else $\left(* \operatorname{sts}_{y}=\operatorname{open}(\cdot, \cdot) \& y \sqsubset x *\right)$

if $\operatorname{prs}_{y}(\varphi)=\perp$ then return $\perp$

else $\left(* \operatorname{prs}_{y}(\varphi)\right.$ is defined $\left.*\right)$

let $\left(z_{1}, \varphi_{1}\right), \ldots,\left(z_{k}, \varphi_{k}\right)$ be all of the pairs in $\operatorname{prs}_{y}(\varphi)$

for $i \longleftarrow 1$ to $k$ do $\Lambda_{i}:=\operatorname{det}-\operatorname{prs}-\operatorname{child}\left(x, z_{i}, \varphi_{i}\right)$

if $\exists j \in\{1, \ldots, k\} . \Lambda_{j}=\perp$ then return $\perp$ else return $\bigcup_{i=1}^{k} \Lambda_{i}$

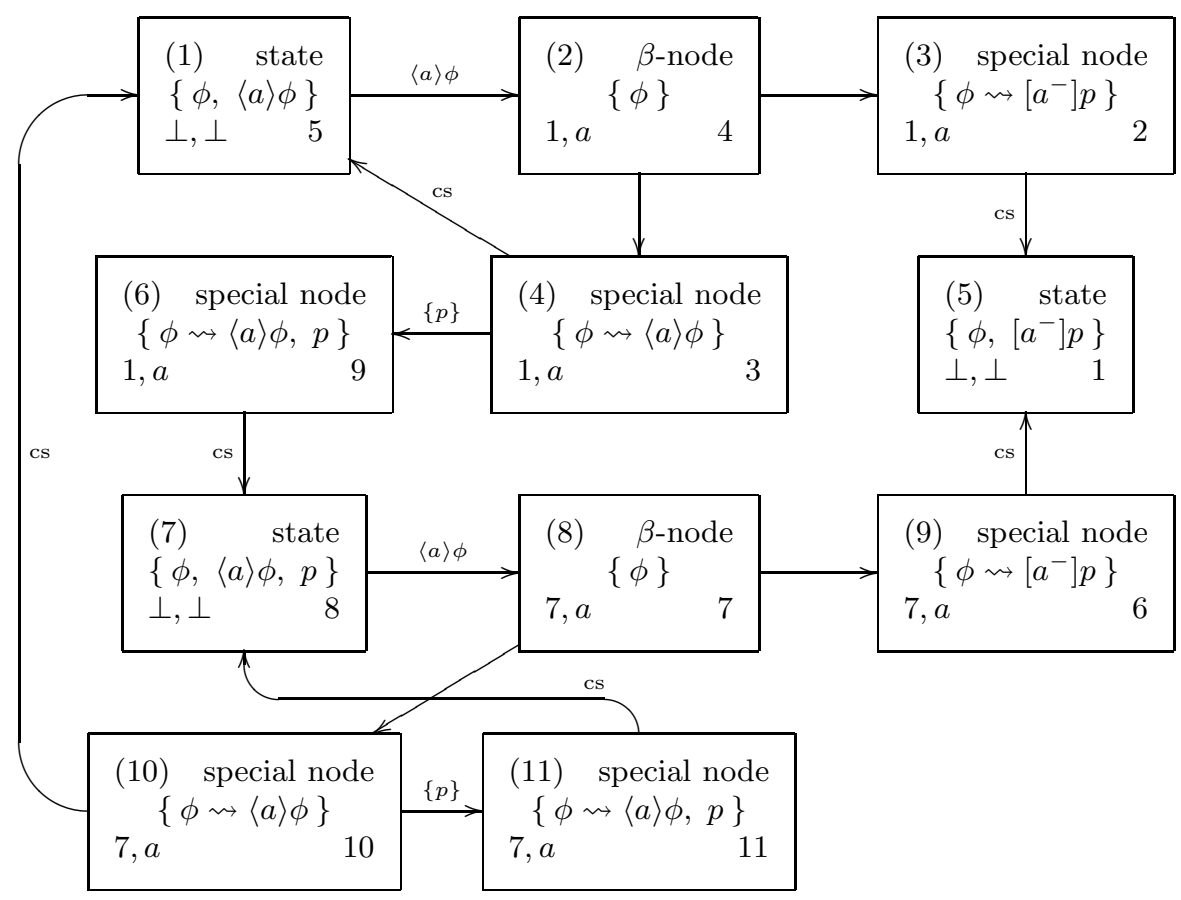

Fig. 1. An example: The graph $G$ just before setting the status of node (2) 


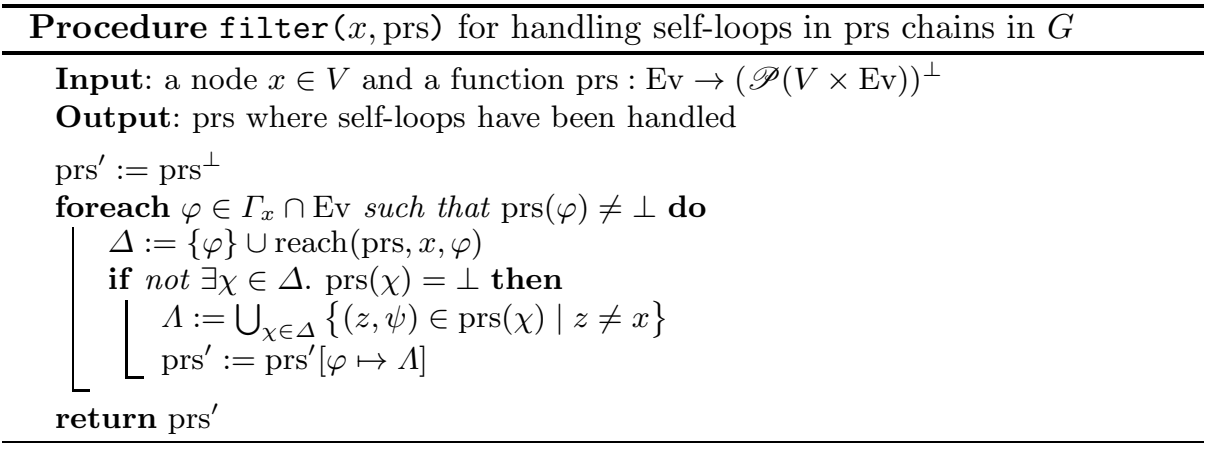

Next we expand (6), which creates (7), then (7), which creates (8), then (8), which creates (9) and (10), and finally (9), which creates no new nodes. Node (9) is similar to (3), but unlike (3), it is compatible with its parent state (7) which results in $\operatorname{sts}_{9}:=$ open $(\perp, \emptyset)$. Using our strategy from the last section, we would now expand (10) so that (8) can become defined after both its children became defined. Since (9) fulfils all its eventualities, we choose to define (8) instead and set $\operatorname{sts}_{8}:=\operatorname{open}(\perp, \emptyset)$. Next we define $(7)$ and then (6) which sets $\operatorname{sts}_{7}:=$ $\operatorname{open}(\perp, \emptyset)$ and $\operatorname{sts}_{6}:=\operatorname{open}(\perp, \emptyset)$. The status of (4) is not affected since (6) was defined after (4), giving “(6) $\not \subset(4)$ ” in det-prs-child(4, 6, $\langle a\rangle \phi)$.

We expand (10) which inserts the edge from (10) to (1). Then we define (10) which creates $(11)$ and sets $\operatorname{sts}_{10}:=\operatorname{open}(\perp, \emptyset)$. Note that the invocation of det-prs-child $(10,1,\langle a\rangle \phi)$ in the invocation det-sts-spl(10) leads to the recursive invocation det-prs-child $(10,6,\langle a\rangle \phi)$. Expanding and defining (11) yields $\operatorname{sts}_{11}:=\operatorname{open}(\perp, \emptyset)$. Finally, no rule is applicable in the shown subgraph.

\section{References}

1. Vardi, M.Y.: The taming of converse: Reasoning about two-way computations. In: Proc. Logic of Programs. Volume 193 of LNCS., Springer (1985) 413-424

2. De Giacomo, G., Massacci, F.: Combining deduction and model checking into tableaux and algorithms for Converse-PDL. Inf. and Comp. 162 (2000) 117-137

3. Nguyen, L.A., Szałas, A.: An optimal tableau decision procedure for Converse-PDL. In: Proc. KSE-09, IEEE Computer Society (2009) 207-214

4. Lange, M., Stirling, C.: Focus games for satisfiability and completeness of temporal logic. In: Proc. LICS-01, IEEE Computer Society (2001) 357-365

5. Lange, M.: Satisfiability and completeness of Converse-PDL replayed. In: Proc. 26th German Conf. on AI (KI-03). Volume 2821 of LNCS., Springer (2003) 79-92

6. Vardi, M., Wolper, P.: Automata theoretic techniques for modal logics of programs. Journal of Computer and System Sciences 32(2) (1986) 183-221

7. Goré, R., Widmann, F.: An optimal on-the-fly tableau-based decision procedure for PDL-satisfiability. In: Proc. CADE-09. Volume 5663 of LNCS. (2009) 437-452

8. Goré, R., Widmann, F.: Sound global state caching for ALC with inverse roles. In: Proc. TABLEAUX-09. Volume 5607 of LNCS., Springer (2009) 205-219 


\section{A Soundness, Completeness, and Complexity}

We start by some more definitions.

Definition 7. We define the size $|\cdot|$ on formulae and programs in negation normal form inductively as follows:

$-|p|:=|\neg p|:=|a|:=\left|a^{-}\right|:=1$

$-|\varphi \wedge \psi|:=|\varphi \vee \psi|:=1+|\varphi|+|\psi|$

$-|\langle\gamma\rangle \varphi|:=|[\gamma] \varphi|:=|\gamma|+|\varphi|$

$-|\gamma ; \delta|:=|\gamma \cup \delta|:=1+|\gamma|+|\delta|$

$-|\varphi ?|:=1+|\varphi|$

$-|\gamma *|:=1+|\gamma|$.

Definition 8. Let $\varphi \in \mathrm{Fml}$ be a formula in negation normal form. The closure $\operatorname{cl}(\varphi)$ is the least set of formulae such that:

$-\varphi \in \operatorname{cl}(\phi)$

- $\langle l\rangle \psi \in \operatorname{cl}(\varphi)$ or $[l] \psi \in \operatorname{cl}(\varphi) \Rightarrow \psi \in \operatorname{cl}(\varphi)$

$-\alpha \in \operatorname{cl}(\varphi) \Rightarrow \alpha_{1} \in \operatorname{cl}(\varphi) \& \alpha_{2} \in \operatorname{cl}(\varphi)$

$-\beta \in \operatorname{cl}(\varphi) \Rightarrow \beta_{1} \in \operatorname{cl}(\varphi) \& \beta_{2} \in \operatorname{cl}(\varphi)$.

It is easy to see that all formula in $\operatorname{cl}(\varphi)$ are also in negation normal form.

Definition 9. A transition frame is a pair $(W, R)$ where $W$ is a non-empty set of worlds and $R: \mathrm{APrg} \rightarrow W \times W$ is a function mapping each atomic program a $\in$ APrg to a binary relation $R_{a}$ over $W$. We extend $R$ to $\operatorname{LPrg}$ by defining $R_{a^{-}}:=$ $\left\{(w, v) \mid(v, w) \in R_{a}\right\}$. A model $(W, R, \mathcal{V})$ is a transition frame $(W, R)$ and a valuation function $\mathcal{V}: \mathrm{AFml} \rightarrow 2^{W}$ mapping each propositional variable $p \in$ $\mathrm{AFml}$ to a set $\mathcal{V}(p)$ of worlds where $p$ is "true".

Definition 10. A formula $\varphi \in \mathrm{Fml}$ is satisfiable iff there exists a model $M=$ $(W, R, \mathcal{V})$ and a world $w \in W$ s.t. $M, w \Vdash \varphi$, and valid iff $\neg \varphi$ is unsatisfiable.

Proposition 11. In the notation of Table 1, the formulae of the form $\alpha \leftrightarrow$ $\alpha_{1} \wedge \alpha_{2}$ and $\beta \leftrightarrow \beta_{1} \vee \beta_{2}$ are valid.

Definition 12. For a given $\varphi \in \mathrm{Fml}$, the (infinite) set $\operatorname{pre}(\varphi)$ is defined as:

$$
\operatorname{pre}(\varphi):=\left\{\psi \in \mathrm{Fml} \mid \exists k \in \mathbb{N}_{0} . \exists \gamma_{1}, \ldots, \gamma_{k} \in \operatorname{Prg} . \psi=\left\langle\gamma_{1}\right\rangle \ldots\left\langle\gamma_{k}\right\rangle \varphi\right\} .
$$

The set of all eventualities is defined as:

$$
\text { Ev }:=\bigcup_{\varphi \in \Delta} \operatorname{pre}(\varphi) \text { where } \Delta:=\{\langle\gamma *\rangle \psi \mid \gamma \in \operatorname{Prg} \& \psi \in \mathrm{Fml}\} .
$$

For all $\varphi, \psi \in \mathrm{Fml}$, the binary relation $\rightsquigarrow$ on formulae is defined as: $\varphi \rightsquigarrow \psi$ iff (exactly) one of the following conditions is true:

- $\exists \chi \in$ Fml. $\exists \gamma, \delta \in \operatorname{Prg} . \varphi=\langle\gamma ; \delta\rangle \chi \& \psi=\langle\gamma\rangle\langle\delta\rangle \chi$ 
$-\exists \chi \in$ Fml. $\exists \gamma, \delta \in$ Prg. $\varphi=\langle\gamma \cup \delta\rangle \chi \&(\psi=\langle\gamma\rangle \chi$ or $\psi=\langle\delta\rangle \chi)$

$-\exists \chi \in \mathrm{Fml} . \exists \gamma \in \operatorname{Prg} . \varphi=\langle\gamma *\rangle \chi \&(\psi=\chi$ or $\psi=\langle\gamma\rangle\langle\gamma *\rangle \chi)$

$-\exists \chi, \phi \in \operatorname{Fml} . \varphi=\langle\phi ?\rangle \chi \& \psi=\chi$.

Intuitively, using Table 1 , the " $\rightsquigarrow$ ” relates a \langle\rangle -formulae $\alpha$ (respectively $\beta$ ), to $\alpha_{1}$ (respectively $\beta_{1}$ and $\beta_{2}$ ) while pre $(\varphi)$ captures that $\langle\gamma *\rangle \varphi$ can be "reduced" to $\langle\gamma\rangle\langle\gamma *\rangle \varphi$, which can be reduced to $\left\langle\gamma_{1}\right\rangle \ldots\left\langle\gamma_{k}\right\rangle\langle\gamma *\rangle$. Note that $\varphi \in \operatorname{pre}(\varphi)$.

Definition 13. A structure $(W, R, L)[$ for $\varphi \in \mathrm{Fml}]$ is a transition frame $(W, R)$ and a labelling function $L: W \rightarrow 2^{\mathrm{Fml}}$ which maps each world $w \in W$ to a set $L(w)$ of formulae [and has $\varphi \in L(v)$ for some world $v \in W$ ].

Definition 14. Let $H=(W, R, L)$ be a structure, $w \in W$, and $\varphi, \psi \in \mathrm{Fml}$. $A$ fulfilling chain for $(H, w, \varphi, \psi)$ is a finite sequence $\left(w_{0}, \psi_{0}\right), \ldots,\left(w_{n}, \psi_{n}\right)$ of world-formula pairs with $n \geq 0$ such that:

- $w_{0}=w, \psi_{0}=\varphi, \psi_{n}=\psi$, and $\psi_{i} \in L\left(w_{i}\right)$ for all $0 \leq i \leq n$

- for all $0 \leq i<n$ : if $\psi_{i}=\langle l\rangle \chi$ for some $l \in \operatorname{LPrg}$ and some $\chi \in \mathrm{Fml}$ then $\psi_{i+1}=\chi$ and $w_{i} R_{b} w_{i+1}$, else $\psi_{i} \rightsquigarrow \psi_{i+1}$ and $w_{i}=w_{i+1}$.

Definition 15. A Hintikka structure $H=(W, R, L)[$ for $\varphi \in \mathrm{Fml}]$ is a structure [for $\varphi]$ which satisfies the following conditions for every $w \in W$, where $\alpha$ and $\beta$ are formulae as defined in Table 1:

$$
\begin{aligned}
& \mathrm{H} 1: \neg p \in L(w) \Rightarrow p \notin L(w) \\
& \mathrm{H} 2: \alpha \in L(w) \Rightarrow \alpha_{1} \in L(w) \& \alpha_{2} \in L(w) \\
& \mathrm{H} 3: \beta \in L(w) \Rightarrow \beta_{1} \in L(w) \text { or } \beta_{2} \in L(w) \\
& \mathrm{H} 4:\langle l\rangle \varphi \in L(w) \Rightarrow \exists v \in W . w R_{b} v \& \varphi \in L(v) \\
& \mathrm{H} 5:[l] \varphi \in L(w) \Rightarrow \forall v \in W . w R_{b} v \Rightarrow \varphi \in L(v) \\
& \mathrm{H} 6:\langle\gamma *\rangle \varphi \in L(w) \Rightarrow \text { there exists a fulfilling chain for }(H, w,\langle\gamma *\rangle \varphi, \varphi) .
\end{aligned}
$$

H3 "locally unwinds" the fix-point semantics of $\langle\gamma *\rangle \varphi$, but does not guarantee a least fix-point which requires $\varphi$ be true eventually. H6 "globally" ensures all $\langle *\rangle$-formulae are fulfilled. H2 captures the greatest fix-point semantics of $[\gamma *] \varphi$.

Proposition 16. A formula $\varphi \in \mathrm{Fml}$ in negation normal form is satisfiable iff there exists a Hintikka structure for $\varphi$.

Proposition 16 implies that we can check whether a formula $\varphi$ in negation normal form is satisfiable by systematically trying to build a Hintikka structure for $\varphi$. Our tableau rules are designed specifically for this purpose.

Definition 17. Let $G=(V, E)$ be a directed graph and $x, y \in V$ two of its nodes. Then $y$ is a child of $x$ iff $(x, y) \in E$. A path $\pi$ in $G$ is a finite or infinite sequence $x_{0}, x_{1}, x_{2}, \ldots$ of nodes in $G$ such that $x_{i+1}$ is a child of $x_{i}$ for all $x_{i}$ except the last node if $\pi$ is finite. An $x$-path $\pi$ is a path in $G$ that has $x_{0}=x$.

We now list some facts about the algorithm which are needed in the subsequent proofs. They can be verified by careful inspection of the procedures. 
Proposition 18. Let $x, y, z \in V$ be nodes and $\varphi, \psi \in \mathrm{Ev}$.

(i) If $x$ is open and $\operatorname{prs}_{x}(\varphi)$ is defined then for each $(y, \psi) \in \operatorname{prs}_{x}(\varphi)$, we have $x \sqsubset y$ and $\psi \in \Gamma_{y}$. Moreover, if $y$ is a state then $\psi$ is a $\langle\mathrm{lp}\rangle$-formula.

(ii) Let $\operatorname{prs}^{\prime}:=$ filter $(x, \operatorname{prs})$. Then $\operatorname{prs}(\varphi)=\operatorname{prs}(\psi) \Rightarrow \operatorname{prs}^{\prime}(\varphi)=\operatorname{prs}^{\prime}(\psi)$.

(iii) The number of consecutive non-states in a path in $G$ is bounded.

(iv) if $x$ and $y$ are states with $\Gamma_{x}=\Gamma_{y}$ then $x=y$;

(v) If $x$ is a state then its parents are exactly the special nodes $y$ with $\Gamma_{y}=\Gamma_{x}$.

(vi) If $x$ is a special node, it has the state $y$ with $\Gamma_{y}=\Gamma_{x}$ as child.

(vii) If $y$ is a child of $x$ and neither of them are states then $\operatorname{pst}_{x}=\operatorname{pst}_{y}$ and $\operatorname{ppr}_{x}=$ $\operatorname{ppr}_{y}$ and $\Gamma_{x} \varsubsetneqq \Gamma_{y}$.

(viii) We have alt $\mathrm{rt}_{\mathrm{rt}}=\emptyset$ and $\emptyset \notin$ alt $_{x}$.

Let $\phi \in \mathrm{Fml}$ be a formula in negation normal form. Furthermore let $G=$ $(V, E)$ be the final graph, with root node rt, which was created by invoking is-sat $(\phi)$. Note that $\Gamma_{\mathrm{rt}}=\{\langle d\rangle \phi\}$ and that all nodes in $G$ are defined once our algorithm terminates. Hence "not closed" then means "open" and vice versa, and $\sqsubset$ is a total strict order. Of course, we have to show first that our algorithm terminates, but this is an immediate consequence from Theorem 19.

Theorem 19. The algorithm runs in EXPTIME in $n:=|\phi|$.

Proof. It is easy to see that every node in $G$ can contain only formulae of the closure $\operatorname{cl}(\phi)$. Furthermore it is known that $|\operatorname{cl}(\phi)| \leq n$ and that $|\varphi| \leq n^{2}$ for all $\varphi \in \operatorname{cl}(\phi)$. Hence there are at most $2^{n}$ different sets of formulae that can be assigned to the nodes of $G$. As a state is uniquely identified by its assigned set of formulae due to Prop. 18(iv), the total number of states in $G$ is also in $2^{\mathcal{O}(n)}$.

If we fix one state $x \in V$, it is not too hard to see that the nodes which are not states and which have $x$ as their parent state form several disjoint trees whose roots are exactly the children of $x$. Because of Prop. 18(vii), the depth of these trees is in $\mathcal{O}(n)$. The branching degree of $\alpha$ - and $\beta$-nodes is constant, the branching degree of a special node, however, is in $2^{\mathcal{O}(n)}$. Hence the size of each tree is in $\left(2^{\mathcal{O}(n)}\right)^{\mathcal{O}(n)} \in 2^{\mathcal{O}\left(n^{2}\right)}$. Since the number of children for each state is clearly in $\mathcal{O}(n)$, the total number of non-states which have $x$ as their parent state is in $\mathcal{O}(n) \cdot 2^{\mathcal{O}\left(n^{2}\right)} \in 2^{\mathcal{O}\left(n^{2}\right)}$. As all non-states have a parent state, we conclude that the total number of nodes in $G$ is in $2^{\mathcal{O}(n)} \cdot 2^{\mathcal{O}\left(n^{2}\right)} \in 2^{\mathcal{O}\left(n^{2}\right)}$.

Bearing that in mind, it is fairly obvious that Rule 1,2 , and 4 can only be applied an exponential number of times. Next we show that Rule 3 can only be applied an exponential number of times. We do this by showing that each node can change from open to open only an exponential number of times.

Assume for the sake of notation that the status of a node $x$ changes from open(alt, prs) to open $\left(\mathrm{alt}^{\prime}, \mathrm{prs}^{\prime}\right)$. In particular we have alt $\neq \mathrm{alt}^{\prime}$ or $\operatorname{prs} \neq \mathrm{prs}^{\prime}$.

First we note that we have alt $\subseteq$ alt ${ }^{\prime}$ which basically follows from the fact that the set of alternative sets of a node is always the union of the sets of alternative sets of its children which are not states, even when the nodes are closed. The only exception are states where the set of alternative sets can become smaller, but only if the node becomes closed. However, all parents of states are special nodes, 
see Prop. 18(iv), and special nodes do not inherit the set of alternative of its corresponding state, see det-sts-spl. As a consequent, alt ${ }_{x}$ can only change an exponential number of times since there are only exponential many alternative sets.

Next we show that if there are $\varphi, \psi \in \operatorname{Ev}$ and $y \in V$ such that either $\operatorname{prs}(\varphi)=$ $\perp \neq \operatorname{prs}^{\prime}(\varphi)$ or $(y, \psi) \in \operatorname{prs}(\varphi)$ but $(y, \psi) \notin \operatorname{prs}^{\prime}(\varphi) \neq \perp$, then some node $z \in V$ must have been closed between the time when prs was calculated and the time when prs $^{\prime}$ was calculated. Hence, there can only be an exponential number of those changes of $\operatorname{prs}_{x}$. Changes of $\operatorname{prs}_{x}$ which do not fall into the described category "fill up" the sets of potential rescuers. Therefore, there can only be an exponential number of such changes of $\operatorname{prs}_{x}$ in a row before one of the described changes must happen. It follows that $\operatorname{prs}_{x}$ can only change an exponential number of times.

To show that some node was closed between the times when $\operatorname{prs}_{x}$ and $\operatorname{prs}_{x}^{\prime}$ were calculated, we use induction on $\sqsubset$. That is, for each node $y$ with $y \sqsubset x$, we can assume the induction hypothesis. When $\operatorname{prs}_{x}$ was calculated via det-sts- $\beta$, det-sts-state, or det-sts-spl, it can be shown - basically because of the "monotonicity" of filter - that there must exist $\varphi_{i}, \psi^{\prime} \in \mathrm{Ev}$ and $y_{i}, y^{\prime} \in V$ such that one of the following statements holds.

- det-prs-child $\left(x, y_{i}, \varphi_{i}\right)=\perp$ at the time of calculating $\operatorname{prs}_{x}$, but we have det-prs-child $\left(x, y_{i}, \varphi_{i}\right) \neq \perp$ at the time of calculating $\operatorname{prs}_{x}^{\prime}$; or

- $\left(y^{\prime}, \psi^{\prime}\right) \in \operatorname{det}-\operatorname{prs}-\operatorname{child}\left(x, y_{i}, \varphi_{i}\right)$ at the time of calculating $\operatorname{prs}_{x}$, but we have $\left(y^{\prime}, \psi^{\prime}\right) \notin \operatorname{det}-\operatorname{prs}-\operatorname{child}\left(x, y_{i}, \varphi_{i}\right) \neq \perp$ at the time of calculating $\operatorname{prs}_{x}^{\prime}$.

We only cover the second case, the first one is similar. Using the definition of det-prs-child, the second case implies one of the following three cases:

Case 1 the node $y_{i}$ was open at the time of calculating $\operatorname{prs}_{x}$, but is closed at the time of calculating $\operatorname{prs}_{x}^{\prime}$;

Case $2 y_{i} \sqsubset x$ and there exists a pair $\left(z_{j}, \varphi_{j}\right)$ such that $\left(z_{j}, \varphi_{j}\right) \in \operatorname{prs}_{y_{i}}\left(\varphi_{i}\right)$ at the time of calculating $\operatorname{prs}_{x}$, but $\left(z_{j}, \varphi_{j}\right) \notin \operatorname{prs}_{y_{i}}\left(\varphi_{i}\right) \neq \perp$ at the time of calculating $\operatorname{prs}_{x}^{\prime}$;

Case $3 y_{i} \sqsubset x$ and there exists a pair $\left(z_{j}, \varphi_{j}\right)$ such that we have $\left(y^{\prime}, \psi^{\prime}\right) \in$ det-prs-child $\left(x, z_{j}, \varphi_{j}\right)$ at the time of calculating $\operatorname{prs}_{x}$, but $\left(y^{\prime}, \psi^{\prime}\right) \notin$ det-prs-child $\left(x, z_{j}, \varphi_{j}\right) \neq \perp$ at the time of calculating $\operatorname{prs}_{x}^{\prime}$.

In Case 1 we have found the node we are looking for, namely $y_{i}$. In Case 2 we can apply the induction hypothesis on $y_{i}$ which gives us the desired node. In Case 2 we can use an inductive argument which is well-defined because of Prop. 18(i). We conclude that each rule of the algorithm can only be applied an exponential number of times.

Next we show that applying a rule can be done in ExpTime. To do this it is obviously enough to show that $\operatorname{det}-\mathbf{s t s}-\beta$, det-sts-state, and det-sts-spl - and hence det-status - run in ExPTIME. It is not too hard to see that the runtime for filter is in ExPTIME. Hence we are left to show that the direct invocations of det-prs-child in $\operatorname{det-sts-} \beta$, det-sts-state, or det-sts-spl 
run in EXPTIME. We have already explained that under each invocation of det-sts- $\beta$, det-sts-state, and det-sts-spl, the procedure det-prs-child is invoked at most once for each pair $(y, \varphi) \in V \times \mathrm{Ev}$ as second and third argument. As there exists only an exponential number of such pairs and the runtime for det-prs-child is clearly in EXPTIME when ignoring recursive invocations, the direct invocations of det-prs-child in det-sts- $\beta$, det-sts-state, or det-sts-spl run in EXPTIME.

Since the number of nodes is exponential and det-status runs in EXPTIME, checking whether one of the rules is applicable can clearly be done in EXPTIME; even with the most naive way of simply trying the conditions for all nodes. We can therefore conclude that the algorithm runs in EXPTIME.

Lemma 20. Let $x \in V$ be an open node.

(i) If $x$ is a state then all of its children are open.

(ii) If $x$ is an $\alpha$-or a $\beta$-node or a special node then some child of $x$ is open.

Proof. Since Rule 3 is not applicable, the node $x$ is up-to-date.

(i): If some child of $x$ were not open then it must be closed. But then $x$ would also be closed by definition of det-sts-state. (ii): If all children of $x$ were not open then all children must be closed. But then $x$ would also be closed by definition of $\operatorname{det}-\mathrm{sts}-\beta$ or det-sts-spl.

Definition 21. Let $x, y \in V$ and $\varphi, \psi \in \mathrm{Fml}$. A graph chain for $(x, \varphi, y, \psi)$ is a finite sequence $\left(y_{0}, \psi_{0}\right), \ldots,\left(y_{n}, \psi_{n}\right)$ of node-formula pairs with $n \geq 0$ such that:

$-y_{0}=x, \psi_{0}=\varphi, y_{n}=y, \psi_{n}=\psi, y_{i}$ is open, and $\psi_{i} \in \Gamma_{y_{i}}$ for all $0 \leq i \leq n$

- $y_{i}=y_{i+1}$ or $y_{i+1}$ is a child of $y_{i}$ for all $0 \leq i<n$

- for all $0 \leq i<n$, (exactly) one of the following conditions is true:

- $\psi_{i}=\psi_{i+1}$ and $\left(y_{i}=y_{i+1}\right.$ or $y_{i}$ is not a state);

- if $\psi_{i}=\langle l\rangle \chi$ for some $l \in \mathrm{LPrg}$ and some $\chi \in \mathrm{Fml}$ then $\psi_{i+1}=\chi$ and $y_{i}$ is a state and $y_{i+1}$ is the successor of $\langle l\rangle \chi$, else $\psi_{i} \rightsquigarrow \psi_{i+1}$ and $y_{i}$ is not a state.

Lemma 22. For every open node $x \in V$ and every eventuality $\varphi \in \Gamma_{x} \cap \mathrm{Ev}$, where $\varphi$ is a $\langle\mathrm{lp}\rangle$-formula if $x$ is a state, we have:

(i) If $\operatorname{prs}_{x}(\varphi)=\perp$ then there exists a node $z \in V$, a formula $\psi \in \mathrm{Fml} \backslash \mathrm{Ev}$ and a graph chain $\sigma$ for $(x, \varphi, z, \psi)$.

(ii) If $\operatorname{prs}_{x}(\varphi) \neq \perp$, we have for all $(z, \psi) \in \operatorname{prs}_{x}(\varphi)$ that there exists a graph chain $\sigma$ for $(x, \varphi, z, \psi)$.

Proof. We use induction on $\sqsubset$. That is, for a node $x \in V$ we can assume that all nodes $y \in V$ with $y \sqsubset x$ fulfil the lemma already. Recall that $y \sqsubset x$ iff $y$ becomes defined before $x$ does.

Let $x \in V$ be open and $\varphi \in \Gamma_{x}$ be an eventuality. We distinguish whether $x$ is an $\alpha / \beta$-node or a state or a special node.

Case 1 (x is $\alpha / \beta$-node): We distinguish whether $\operatorname{prs}_{x}(\varphi) \neq \perp$ or $\operatorname{prs}_{x}(\varphi)=\perp$. 
Case $1.1\left(\operatorname{prs}_{x}(\varphi) \neq \perp\right)$ : Let $(z, \psi) \in \operatorname{prs}_{x}(\varphi)$. We distinguish whether or not there exists an open child $y:=y_{i}$ of $x$ such that $(z, \psi)$ is an element of the corresponding $\Lambda_{\varphi, i}$ in det-sts- $\beta(x)$.

Case 1.1.1: (y exists) In this case $(z, \psi)$ must be in det-prs-child $(x, y, \varphi)$. Our final case distinction is whether or not $y \sqsubset x$.

Case 1.1.1.1: (not $y \sqsubset x$ ) In this case, we have $y=z$ and $\varphi=\psi$ by construction in det-prs-child. Hence $(x, \varphi),(y, \varphi)$ is a graph chain for $(x, \varphi, z, \psi)$.

Case 1.1.1.2: $(y \sqsubset x)$ In this case, we have $\operatorname{prs}_{y}(\varphi) \neq \perp$ and there must exist a pair $\left(y^{\prime}, \varphi^{\prime}\right) \in \operatorname{prs}_{y}(\varphi)$ such that $(z, \psi)$ is contained in det-prs-child $\left(x, y^{\prime}, \varphi^{\prime}\right)$. Using the induction hypothesis on $y$, we obtain a graph chain $\sigma_{1}$ for $\left(y, \varphi, y^{\prime}, \varphi^{\prime}\right)$. In particular $y^{\prime}$ is open and we have $y \sqsubset y^{\prime}$ by Prop. 18(i). We can now inductively repeat the same arguments for $y^{\prime}$ and $\varphi^{\prime}$ that we have used for $y$ and $\varphi$. Since $y \sqsubset y^{\prime}$, we must eventually end up in Case 1.1.1.1. Hence the induction is well-defined and yields a graph chain $\sigma_{2}$ for $\left(y^{\prime}, \varphi^{\prime}, z, \psi\right)$. Thus $(x, \varphi), \sigma_{1}, \sigma_{2}$ is a graph chain for $(x, \varphi, z, \psi)$.

Case 1.1.2: ( $y$ does not exist) In this case we know that $(z, \psi) \notin \operatorname{prs}(\varphi)$ in $\operatorname{det-sts-} \beta(x)$ but must have been inserted in filter( $x$, prs), which is invoked at the end of det-sts- $\beta(x)$. Hence there exists a $\chi \in \operatorname{reach}(\operatorname{prs}, x, \varphi)$ such that $(z, \psi) \in \operatorname{prs}(\chi)$. According to the definition of reach there exist $\varphi_{0}, \ldots, \varphi_{k} \in$ Ev such that $\chi=\varphi_{k}$ and $\left(x, \varphi_{0}\right) \in \operatorname{prs}(\varphi)$ and $\left(x, \varphi_{i+1}\right) \in \operatorname{prs}\left(\varphi_{i}\right)$ for all $0 \leq$ $i<k$. Since $\left(x, \varphi_{0}\right) \in \operatorname{prs}(\varphi)$ there exists an open child $y_{i}$ such that $\left(x, \varphi_{0}\right)$ is an element of the corresponding $\Lambda_{\varphi, i}$ in det-sts- $\beta(x)$. Hence we can obtain a graph chain $\sigma_{-1}$ for $\left(x, \varphi, x, \varphi_{0}\right)$ exactly as in Case 1.1.1. Using the same arguments, we can also get graph chains $\sigma_{i}$ for $\left(x, \varphi_{i}, x, \varphi_{i+1}\right)$ for all $0 \leq i<k$, and a graph chain $\sigma_{k}$ for $(x, \chi, z, \psi)$. Because we have $\chi=\varphi_{k}$, their concatenation $\sigma_{0}, \ldots, \sigma_{k}$ is a graph chain for $(x, \varphi, z, \psi)$.

Case 1.2 $\left(\operatorname{prs}_{x}(\varphi)=\perp\right)$ : We distinguish whether or not there exists an open child $y:=y_{i}$ of $x$ such that the corresponding set $\Lambda_{\varphi, i}$ in $\operatorname{det-sts-} \beta(x)$ is undefined.

Case 1.2.1: ( $y$ exists) In this case det-prs-child $(x, y, \varphi)$ must return undefined. Hence we must have $y \sqsubset x$ by construction in det-prs-child. Our final case distinction is whether or not $\operatorname{prs}_{y}(\varphi)=\perp$.

Case 1.2.1.1: $\left(\operatorname{prs}_{y}(\varphi)=\perp\right)$ In this case then we can use the induction hypothesis on $y$ to get a graph chain $\sigma$ for $(y, \varphi, z, \psi)$ for some $z \in V$ and some $\psi \in \operatorname{Fml} \backslash \mathrm{Ev}$. Thus $(x, \varphi), \sigma$ is a graph chain for $(x, \varphi, z, \psi)$.

Case 1.2.1.2: $\left(\operatorname{prs}_{y}(\varphi) \neq \perp\right)$ In this case there must exist a $\left(y^{\prime}, \varphi^{\prime}\right) \in \operatorname{prs}_{y}(\varphi)$ such that det-prs-child $\left(x, y^{\prime}, \varphi^{\prime}\right)$ returns undefined. Using the second part of the induction hypothesis on $y$, we obtain a graph chain $\sigma_{1}$ for $\left(y, \varphi, y^{\prime}, \varphi^{\prime}\right)$. In particular $y^{\prime}$ is open and we have $y \sqsubset y^{\prime}$ by Prop. 18(i). We can now inductively repeat the same arguments for $y^{\prime}$ and $\varphi^{\prime}$ that we have used for $y$ and $\varphi$. Since $y \sqsubset$ $y^{\prime}$, we must eventually end up in Case 1.1.2.1. Hence the induction is well-defined and yields a graph chain $\sigma_{2}$ for $\left(y^{\prime}, \varphi^{\prime}, z, \psi\right)$ for some $z \in V$ and some $\psi \in$ Fml $\backslash$ Ev. Thus $(x, \varphi), \sigma_{1}, \sigma_{2}$ is a graph chain for $(x, \varphi, z, \psi)$.

Case 1.2.2: ( $y$ does not exist) In this case we know that $\operatorname{prs}(\varphi)$ in det-sts- $\beta(x)$ is defined. Therefore $\operatorname{prs}_{x}(\varphi)$ became undefined in filter $(x, \operatorname{prs})$, which is in- 
voked at the end of det-sts- $\beta(x)$. Hence there exists a $\chi \in \operatorname{reach}(\operatorname{prs}, x, \varphi)$ such that $\operatorname{prs}(\chi)=\perp$. According to the definition of reach there exist $\varphi_{0}, \ldots, \varphi_{k} \in \mathrm{Ev}$ such that $\chi=\varphi_{k}$ and $\left(x, \varphi_{0}\right) \in \operatorname{prs}(\varphi)$ and $\left(x, \varphi_{i+1}\right) \in \operatorname{prs}\left(\varphi_{i}\right)$ for all $0 \leq i<k$. Since $\left(x, \varphi_{0}\right) \in \operatorname{prs}(\varphi)$, there exists an open child $y:=y_{i}^{\prime}$ such that $\left(x, \varphi_{0}\right)$ is an element of the corresponding $\Lambda_{\varphi, i}$ in det-sts- $\beta\left(x, y_{i}^{\prime}\right)$. Hence we can obtain a graph chain $\sigma_{-1}$ for $\left(x, \varphi, x, \varphi_{0}\right)$ exactly as in Case 1.1.1. Using the same arguments, we can also get graph chains $\sigma_{i}$ for $\left(x, \varphi_{i}, x, \varphi_{i+1}\right)$ for all $0 \leq i<$ $k$. Since $\operatorname{prs}(\chi)=\perp$, there exists an open child $y:=y_{j}^{\prime}$ such that the corresponding $\Lambda_{\varphi, j}$ in det-sts- $\beta(x)$ is undefined. Thus we can obtain a graph chain $\sigma_{k}$ for $(x, \chi, z, \psi)$ for some $z \in V$ and some $\psi \in \mathrm{Fml} \backslash$ Ev exactly as in Case 1.2.1. Because we have $\chi=\varphi_{k}$, the concatenation $\sigma_{0}, \ldots, \sigma_{k}$ is a graph chain for $(x, \varphi, z, \psi)$.

Case 2 ( $x$ is a state): By assumption $\varphi$ is of the form $\langle l\rangle \chi$ for some $l \in \mathrm{LPrg}$ and some $\chi \in \mathrm{Ev}$. By distinguishing whether or not $\operatorname{prs}_{x}(\langle l\rangle \chi)=\perp$, we obtain the appropriate graph chain almost exactly as in Case 1. The main difference is that we have only one child of $x$ to consider, namely the successor of $\langle l\rangle \chi$.

Case 3 ( $x$ is a special node): First we inductively build a sequence $\sigma_{0}$ as follows. We start with $\sigma_{0}:=(x, \varphi)$. If $\varphi$ is a $\langle\mathrm{lp}\rangle$-formula then we stop. Otherwise we extend $\sigma_{0}$ with $\varphi^{\prime}:=\operatorname{ann}_{x}(\varphi)$ which must be defined as $x$ is a special node and $\operatorname{ann}_{x}$ is hence a full annotation. Remember that we have $\varphi \rightsquigarrow \varphi^{\prime}$. If $\varphi^{\prime}$ is not an eventuality nor a $\langle\mathrm{lp}\rangle$-formula then we stop. Otherwise we extend $\sigma_{0}$ with $\varphi^{\prime \prime}:=\operatorname{ann}_{x}\left(\varphi^{\prime}\right)$ and so on. The termination of this iteration is guaranteed by the fact that $x$ is open and hence $\operatorname{ann}_{x}$ is non-cyclic. It is not hard to see that the final $\sigma_{0}$ is of the form $(x, \varphi),\left(x, \varphi^{\prime}\right), \ldots,(x, \chi)$ where $\chi=\operatorname{defer}(x, \varphi)$. Hence we have $\operatorname{prs}_{x}(\varphi)=\operatorname{prs}_{x}(\chi)$ by definition of det-sts-state and because of Prop. 18(ii). If $\chi \notin \mathrm{Ev}$ then we know $\operatorname{prs}_{x}(\chi)=\operatorname{prs}_{x}(\varphi)=\perp$. Furthermore $\sigma_{0}$ is a graph chain for $(x, \varphi, x, \chi)$ and we are done. Otherwise $\chi$ is of the form $\langle l\rangle \chi^{\prime}$ for some $l \in \operatorname{LPrg}$ and some $\chi^{\prime} \in \mathrm{Ev}$.

By distinguishing whether or not $\operatorname{prs}_{x}\left(\langle l\rangle \chi^{\prime}\right)=\perp$, we obtain the appropriate graph chain $\sigma_{1}$ for $\left(x,\langle l\rangle \chi^{\prime}, z, \psi\right)$ exactly as in Case 1. Note in particular that we can use the induction hypothesis on the child of $x$ which is a state since our eventuality is of the form $\langle l\rangle \chi^{\prime}$. We can then conclude that $\sigma_{0}, \sigma_{1}$ is the appropriate graph chain for $(x, \varphi, z, \psi)$.

Definition 23. For $\varphi \in \mathrm{Ev}$, let $\operatorname{ex}(\varphi) \in \mathrm{Fml}$ be the largest subformula of $\varphi$ such that $\operatorname{ex}(\varphi) \notin \operatorname{Ev}$ and $\varphi \in \operatorname{pre}(\operatorname{ex}(\varphi))$.

It is easy to see that $\operatorname{ex}(\varphi)$ is well-defined, in particular, that it is unique.

Proposition 24. Let $\psi_{0}, \ldots, \psi_{n}$ be a finite sequence of formulae with $n \geq 0$ such that $\psi_{n} \notin \mathrm{Ev}$ and for every $0 \leq i \leq n$ : if $\psi_{i}=\langle l\rangle \chi$ for some $l \in \operatorname{LPrg}$ and some $\chi \in \mathrm{Fml}$ then $\psi_{i+1}=\chi$, else $\psi_{i} \rightsquigarrow \psi_{i+1}$.

(i) $\operatorname{ex}\left(\psi_{j}\right)=\psi_{n}$ for every $0 \leq j \leq n$;

(ii) for every $\chi \in \mathrm{Fml}$ such that $\psi_{n}$ is a subformula of $\chi$ and $\chi$ is a subformula of $\psi_{0}$, there exists $a 0 \leq j \leq n$ such that $\chi=\psi_{j}$.

Theorem 25. If root $\mathrm{rt} \in V$ is open then $\phi$ is satisfiable. 
Proof. We will show that there exists a Hintikka structure for $\phi$. The theorem then follows with Prop. 16.

Let $G_{\mathrm{p}}$ ("p" for pruned) be the subgraph of $G$ that consists of all open nodes of $G$ which are not states, and all open states for which there exists a full noncyclic annotation. Note that open states which are children of open special nodes are contained in $G_{\mathrm{p}}$ since the annotation of the special child is trivially a full noncyclic annotation for the state. The edges of $G_{\mathrm{p}}$ are exactly the edges of $G$ which connect two open nodes. Then we use $G_{\mathrm{p}}$ to generate a structure $H=(W, R, L)$ as described next.

Let $W$ be the set of all states of $G_{\mathrm{p}}$. To define $R$ we first define the auxiliary function $R: \operatorname{LPrg} \rightarrow W \times W$. For every $l \in \operatorname{LPrg}$ and every $s, t \in W$, let $(s, t) \in$ $R^{\prime}(b)$ iff $\Gamma_{s}$ contains a formula $\langle l\rangle \varphi$ for some formula $\varphi \in \mathrm{Fml}$ and there exists an $s$-path $x_{0}=s, x_{1}, \ldots, x_{k+1}, x_{k+2}=t$ in $G_{\mathrm{p}}$ such that $x_{1}$ is the successor of $\langle l\rangle \varphi$, each $x_{i}, 1 \leq i \leq k$, is an $\alpha$ - or a $\beta$-node or a special node, and $x_{k+1}$ is a special node. Thus state $t$ is a "saturation" of $x_{1}$. Then we set $R_{a}:=R^{\prime}(a) \cup\{(t, s) \mid$ $\left.(s, t) \in R^{\prime}\left(a^{-}\right)\right\}$for every $a \in \operatorname{APrg}$. Finally, we set $L(s)$ to be $\Gamma_{s}$ for all $s \in W$.

As rt is open, the only child $y$ of rt is also open due to Lemma 20(i). Moreover we have $\phi \in \Gamma_{y}$ because $y$ is the successor of $\langle d\rangle \phi \in \Gamma_{\text {rt }}$. Thus there exists an open state $s$ in $G$, and thus in $G_{\mathrm{p}}$, such that $\phi \in \Gamma_{s}=L(s)$ due to Lemma 20(ii) and Prop. 18(iii)(v)(vii). Hence $H$ is a structure for $\phi$.

Next we show that $H$ fulfils $\mathrm{H} 1$ to $\mathrm{H} 6$ and is thus a Hintikka structure for $\phi$. Let $w \in W$, that is $w$ is a state in $G_{\mathrm{p}}$. In particular $w$ is open.

H1: The set $L(w)=\Gamma_{w}$ cannot contain a contradiction as $w$ is not closed.

$\mathrm{H} 2$ and $\mathrm{H} 3$ follow from the fact that $w$ is a state and not an $\alpha$ - or a $\beta$-node.

H4 follows from the treatment of states in expand, Lemma 20(ii) as well as Prop. 18(iii)(v)(vii).

H5: Let $[l] \varphi \in L(w)$ and $v \in W$ such that $(w, v) \in R_{b}$. We distinguish whether $(w, v) \in R^{\prime}(b)$ or $(v, w) \in R^{\prime}\left(b^{\smile}\right)$.

If $(w, v) \in R^{\prime}(b)$, we have by definition of $R^{\prime}$ that $\Gamma_{w}$ contains a formula $\langle l\rangle \psi$ for some $\psi \in \mathrm{Fml}$ and there exists a path $x_{0}=w, x_{1}, \ldots, x_{k+1}, x_{k+2}=v$ in $G_{\mathrm{p}}$ such that $x_{1}$ is the successor of $\langle l\rangle \psi$, each $x_{i}, 1 \leq i \leq k$, is an $\alpha$ - or a $\beta$-node or a special node, and $x_{k+1}$ is a special node. By construction we have $\varphi \in \Gamma_{x_{1}}$, and by Prop. 18(v) and (vii) we have $\varphi \in \Gamma_{v}=L(v)$.

If $(v, w) \in R^{\prime}\left(b^{\smile}\right)$ then we have by definition of $R^{\prime}$ that the set $\Gamma_{v}$ contains a formula $\left\langle b^{\smile}\right\rangle \psi$ for some $\psi \in \mathrm{Fml}$, and that there exists a path $x_{0}=$ $v, x_{1}, \ldots, x_{k+1}, x_{k+2}=w$ in $G_{\mathrm{p}}$ such that $x_{1}$ is the successor of $\left\langle b^{\smile}\right\rangle \psi$, and each $x_{i}, 1 \leq i \leq k$, is an $\alpha$ - or a $\beta$-node or a special node, and $x_{k+1}$ is a special node. By construction and Prop. 18(vii) we have pst $_{x_{k+1}}=v$ and $\operatorname{ppr}_{x_{k+1}}=b^{\smile}$. Together with Prop. 18(vi) and the fact that $\left(r^{\smile}\right)^{\smile}=r$ and that $x_{k+1}$ is not closed, we can therefore conclude $\varphi \in \Gamma_{v}=L(v)$.

H6: We use Lemma 22 as is shown next. Let $\varphi \in L(w)=\Gamma_{w}$ be an eventuality. We first build a graph chain $\sigma$ for $(w, \varphi, y, \psi)$ for some $y \in V$ and some $\psi \in$ Fml $\backslash \mathrm{Ev}$ and then convert $\sigma$ into a fulfilling chain.

By assumption there exists a full non-cyclic annotation ann for $w$. First we inductively build a sequence $\sigma_{0}$ as follows. We start with $\sigma_{0}:=(w, \varphi)$. If $\varphi$ is a $\langle\operatorname{lp}\rangle$ - 
formula then we stop. Otherwise we extend $\sigma_{0}$ with $\varphi^{\prime}:=\operatorname{ann}(\varphi)$ which must be defined as ann is a full annotation. Remember that we have $\varphi \rightsquigarrow \varphi^{\prime}$. If $\varphi^{\prime}$ is not an eventuality nor a $\langle$ lp $\rangle$-formula then we stop. Otherwise we extend $\sigma_{0}$ with $\varphi^{\prime \prime}:=$ $\operatorname{ann}\left(\varphi^{\prime}\right)$ and so on. The termination of this iteration is guaranteed by the fact that ann is non-cyclic. Let the final $\sigma_{0}$ be of the form $(w, \varphi),\left(w, \varphi^{\prime}\right), \ldots,(w, \chi)$. If $\chi \notin \mathrm{Ev}$ then $\sigma_{0}$ is already a graph chain for $(w, \varphi, w, \chi)$. and we set $\sigma:=\sigma_{0}$. Otherwise $\chi$ is of the form $\langle l\rangle \chi^{\prime}$ for some $l \in \operatorname{LPrg}$ and some $\chi^{\prime} \in \mathrm{Ev}$. In this case we build another graph chain $\sigma_{1}$ for $(w, \chi, y, \psi)$ for some $y \in V$ and some $\psi \in \mathrm{Fml} \backslash \mathrm{Ev}$ by distinguishing whether or not $\operatorname{prs}_{w}(\chi)=\perp$, and then set $\sigma:=\sigma_{0}, \sigma_{1}$.

Case 1: If $\operatorname{prs}_{w}(\chi)=\perp$ then Lemma 22 provides us with a graph chain $\sigma_{1}$ for $(w, \chi, y, \psi)$ for some $y \in V$ and some $\psi \in \mathrm{Fml} \backslash \mathrm{Ev}$.

Case 2: If $\operatorname{prs}_{w}(\varphi) \neq \perp$ then it contains at least one pair $(y, \psi)$ such that $w \sqsubset$ $y$ because of Prop. 18(i) and the fact that Rule 4 is not applicable. Hence Lemma 22 gives us a graph chain for $(w, \chi, y, \psi)$. In particular $y$ is open. As the $\sqsubset$-relation is well-founded, we can inductively apply Lemma 22 on $y$ and $\psi$ and so on, and must eventually encounter Case 1. Joining all sequences in the obvious way gives us a graph chain $\sigma$ for $(w, \chi, y, \psi)$ for some $y \in V$ and some $\psi \in \mathrm{Fml} \backslash \mathrm{Ev}$.

To convert $\sigma$ into a fulfilling chain, we extend $\sigma$ as follows. Let $\pi=z_{0}, \ldots, z_{k}$ be a finite $y$-path in $G_{\mathrm{p}}$ such that $t:=z_{k}$ is the only state in $\pi$ and $\psi \in$ $\Gamma_{z_{i}}$ for all $0 \leq i \leq k$. The existence of $\pi$ follows from Lemma 20(ii) and Prop. 18(iii)(v)(vii). Note that $\pi=y$ iff $y$ is a state. We extend $\sigma$ by the sequence $\left(z_{1}, \psi\right), \ldots,\left(z_{k}, \psi\right)$. Now $\sigma$ is a graph chain for $(w, \varphi, t, \psi)$.

Finally, we convert $\sigma$ into a fulfilling chain as follows. Let $\sigma$ be of the form $\left(y_{0}, \psi_{0}\right), \ldots,\left(y_{n}, \psi_{n}\right)$. Next we replace each $y_{i}, 0 \leq i \leq n$ in $\sigma$ with the first state which appears on the path $y_{i}, \ldots, y_{n}$. Furthermore we contract all consecutive repetitions of pairs. Let the resulting $\sigma$ be of the form $\left(w_{0}, \psi_{0}^{\prime}\right), \ldots,\left(w_{m}, \psi_{m}^{\prime}\right)$.

It is not too hard to check that $\sigma$ is a fulfilling chain for $(H, w, \varphi, \psi)$. Moreover, Prop. 24(i) tells us that $\psi=\operatorname{ex}(\varphi)$. If $\varphi=\langle\gamma *\rangle \chi$ for some $\gamma \in \operatorname{Prg}$ and some $\chi \in \mathrm{Fml}$ then $\operatorname{ex}(\varphi)$ is a subformula of $\chi$ which is obviously a subformula of $\varphi$. According to Prop. 24(ii), there therefore exists a $j \in\{0, \ldots, m\}$ such that $\chi=\psi_{j}^{\prime}$. Thus $\left(w_{0}, \psi_{0}^{\prime}\right), \ldots,\left(w_{j}, \psi_{j}^{\prime}\right)$ is a fulfilling chain for $(H, w,\langle\gamma *\rangle \chi, \chi)$. Hence $\mathrm{H} 6$ holds which concludes the proof.

We next define some concepts related to models and state some propositions which we will need in the remaining proofs.

Definition 26. Let $M=(W, R, \mathcal{V})$ be a model, $w \in W$, and $\varphi, \psi \in$ Fml. $A$ model chain for $(M, w, \varphi, \psi)$ is a finite sequence $\left(w_{0}, \psi_{0}\right), \ldots,\left(w_{n}, \psi_{n}\right)$ of worldformula pairs with $n \geq 0$ such that:

(i) $w_{0}=w, \psi_{0}=\varphi, \psi_{n}=\psi$, and $M, w_{i} \Vdash \psi_{i}$ for all $0 \leq i \leq n$

(ii) for all $0 \leq i<n$ : if $\psi_{i}=\langle l\rangle \chi$ for some $l \in \operatorname{LPrg}$ and some $\chi \in \mathrm{Fml}$ then $\psi_{i+1}=\chi$ and $w_{i} R_{b} w_{i+1}$, else $\psi_{i} \rightsquigarrow \psi_{i+1}$ and $w_{i}=w_{i+1}$.

Proposition 27. Let $M=(W, R, \mathcal{V})$ be a model, $w \in W$, and $\varphi, \psi \in \mathrm{Fml}$ such that $\varphi \in \operatorname{pre}(\psi)$ and $M, w \Vdash \varphi$. Then there exists a model chain for $(M, w, \varphi, \psi)$. 
Definition 28. Let $X$ and $Y$ be sets and $f, g: X \rightarrow Y^{\perp}$ be functions. We say that $g$ extends $f$ iff $\forall x \in X . f(x) \neq \perp \Rightarrow g(x)=f(x)$.

Let $\operatorname{Seq}(X)$ be the set of non-empty, finite sequences of elements in $X$. For a sequence $\sigma=x_{0}, \ldots, x_{n}$ in $\operatorname{Seq}(X)$, let $|\sigma|:=n+1$ be the length of $\sigma$. Furthermore, for all $0 \leq i<|\sigma|$, we define $\sigma_{i}:=x_{i}$ and $\sigma_{\geq i}:=x_{i}, x_{i+1}, \ldots, x_{n}$.

Definition 29. Let $M=(W, R, \mathcal{V})$ be a model, $w \in W$, and $\Gamma \subseteq$ Fml. We write $M, w \Vdash \Gamma$ iff $M, w \Vdash \varphi$ for all $\varphi \in \Gamma$. Furthermore, let ann $: \overline{\mathrm{Ev}} \rightarrow \mathrm{Fml}^{\perp}$ be an annotation for $\Gamma$ and $\mathrm{chn}: \mathrm{Ev} \rightarrow(\mathrm{Seq}(W \times \mathrm{Fml}))^{\perp}$ a function. We say that $(M, w, \mathrm{chn})$ annotated-satisfies $(\Gamma$, ann $)$ iff the following conditions hold:

$-M, w \Vdash \Gamma$

$-\operatorname{chn}(\varphi)$ is defined for all $\varphi \in \Gamma \cap \mathrm{Ev}$

- for all $\varphi \in \mathrm{Ev}$ such that $\sigma:=\operatorname{chn}(\varphi)$ is defined, we have:

- the sequence $\sigma$ is a model chain for $(M, w, \varphi, \operatorname{ex}(\varphi))$

- for all $0 \leq i<|\sigma|$, if $\sigma_{i}$ is of the form $(w, \psi)$ for some $\psi \in \operatorname{Ev}$ then $\perp \neq$ $\operatorname{chn}(\psi)=\sigma_{\geq i}$

- if $\operatorname{ann}(\varphi) \neq \perp$ then $\sigma_{1}=(w, \operatorname{ann}(\varphi))$.

If there exists such a triple $(M, w, \mathrm{chn})$ we call $(\Gamma$, ann) annotated-satisfiable.

Proposition 30. Let $M=(W, R, \mathcal{V})$ be a model, $w \in W, \varphi \in \mathrm{Fml}$, and $\Gamma \subseteq$ Fml be a finite set, such that $M, w \Vdash \varphi$ and $M, w \Vdash \Gamma$.

(i) Let ann : $\mathrm{Ev} \rightarrow \mathrm{Fml}^{\perp}$ be an annotation for $\Gamma$ and $\operatorname{chn}: \mathrm{Ev} \rightarrow(\operatorname{Seq}(W \times$ $\mathrm{Fml}))^{\perp}$ a function such that $(M, w, \mathrm{chn})$ annotated-satisfies $(\Gamma, \mathrm{ann})$. If we have $\varphi \notin \mathrm{Ev}$ or $\operatorname{ann}(\varphi)=\perp$ then there exists an extension chn' $^{\prime}$ of chn such that $\left(M, w, \mathrm{chn}^{\prime}\right)$ annotated-satisfies $(\Gamma \cup\{\varphi\}$, ann $)$.

(ii) Let $\sigma$ be a model chain for $(M, w, \varphi, \operatorname{ex}(\varphi))$ such that $\sigma_{i} \neq \sigma_{j}$ for all $0 \leq$ $i, j<|\sigma|, i \neq j$. Then there exists a function $\operatorname{chn}^{\prime}: \operatorname{Ev} \rightarrow(\operatorname{Seq}(W \times \mathrm{Fml}))^{\perp}$ such that $\left(M, w, \operatorname{chn}^{\prime}\right)$ annotated-satisfies $\left(\Gamma \cup\{\varphi\}, \operatorname{ann}^{\perp}\right)$ and $\operatorname{chn}^{\prime}(\varphi)=\sigma$ if $\varphi \in \mathrm{Ev}$.

For a node $x \in V$, we say that $(M, w, \operatorname{chn})$ annotated-satisfies $x$ iff $(M, w, \operatorname{chn})$ annotated-satisfies $\left(\Gamma_{x}, \operatorname{ann}_{x}\right)$. Similarly, we say that $x$ is annotated-satisfiable iff $\left(\Gamma_{x}, \operatorname{ann}_{x}\right)$ is annotated-satisfiable.

Definition 31. Let $M=(W, R, \mathcal{V})$ be a model, $w, v \in W$, and $\operatorname{chn}: \mathrm{Ev} \rightarrow$ $(\mathrm{Seq}(W \times \mathrm{Fml}))^{\perp}$ be a function.

- Let $x \in V$ be a state. We say that $(M, w)$ realises $x$ iff $M, w \Vdash \Gamma_{x}$ and $M, w \nVdash$ $\left(\Gamma_{x} \cup \Gamma\right)$ for all $\Gamma \in$ alt $_{x}$.

- Let $x$ be an $\alpha$ - or a $\beta$-node or a special node. We say that $(M, v, w, \operatorname{chn})$ realises $x$ iff $M, v \Vdash \Gamma_{\mathrm{pst}_{x}}, M, v \nVdash\left(\Gamma_{\mathrm{pst}_{x}} \cup \Gamma\right)$ for all $\Gamma \in \operatorname{alt}_{x}, v R_{\mathrm{ppr}_{x}} w$ and $(M, w, \mathrm{chn})$ annotated-satisfies $x$.

We call a node $x \in V$ realisable iff there exists an appropriate tuple $(M, w)$ or $(M, v, w, \mathrm{chn})$ which realises $x$. 
Lemma 32. Let $M=(W, R, \mathcal{V})$ be a model, $w, v \in W$, and $\operatorname{chn}: \mathrm{Ev} \rightarrow$ $(\mathrm{Seq}(W \times \mathrm{Fml}))^{\perp}$ be a function,

(i) Let $x \in V$ be an $\alpha$-or a $\beta$-node such that $x$ is up-to-date and $(M, v, w, \mathrm{chn})$ realises $x$. Then there exists a child $y$ of $x$ and an extension chn' $^{\prime}$ of chn such that $\left(M, v, w, \mathrm{chn}^{\prime}\right)$ realises $y$.

(ii) Let $x \in V$ be a state such that $(M, w)$ realises $x$. Moreover, let $\langle l\rangle \psi \in \Gamma_{x}$ and $y \in V$ be the successor of $\langle l\rangle \psi$ such that alt $_{y} \subseteq$ alt $_{x}$. Furthermore, let $\sigma$ be a model chain for $(M, w,\langle l\rangle \psi, \operatorname{ex}(\langle l\rangle \psi))$ such that $\sigma_{i} \neq \sigma_{j}$ for all $0 \leq$ $i, j<|\sigma|, i \neq j$, and let $v^{\prime} \in W$ such that $\sigma_{1}=\left(v^{\prime}, \psi\right)$. Then there exists a function $\operatorname{chn}^{\prime}: \mathrm{Ev} \rightarrow(\mathrm{Seq}(W \times \mathrm{Fml}))^{\perp}$ such that $\left(M, w, v^{\prime}, \operatorname{chn}^{\prime}\right)$ realises $y$ and $\operatorname{chn}^{\prime}(\psi)=\sigma_{\geq 1}$ if $\psi \in \mathrm{Ev}$.

(iii) Let $x \in V$ be a special node such that $x$ is up-to-date and ( $M, v, w, \operatorname{chn})$ realises $x$. Then there either exists a child $y$ of $x$ and an extension chn' of chn such that $y$ is not a state and $\left(M, v, w, \mathrm{chn}^{\prime}\right)$ realises $y$, or $(M, w)$ realises $\operatorname{get}$ Child $(x, \mathrm{cs})$.

Proof. (i): We distinguish whether or not the principal formula $\varphi \in \Gamma_{x}$, which is decomposed in $x$, is an eventuality.

If $\varphi \in \operatorname{Ev}$ then $\sigma:=\operatorname{chn}(\varphi)$ is a model chain for $(M, w, \varphi, \operatorname{ex}(\varphi))$. Since $\varphi$ is not a $\langle$ lp $\rangle$-formula, we therefore have $\sigma_{1}=\left(w, \varphi^{\prime}\right)$ for some $\varphi^{\prime} \in \mathrm{Fml}$ such that $\varphi \rightsquigarrow \varphi^{\prime}$ and $M, w \Vdash \varphi^{\prime}$. Due to the definition of $\rightsquigarrow$ and expand, there exists a child $y \in V$ of $x$ such that $\Gamma_{y}=\Gamma_{x} \uplus\left\{\varphi^{\prime}\right\}$ and $\operatorname{ann}_{y}=\operatorname{ann}_{x}\left[\varphi \mapsto \varphi^{\prime}\right]$. Together with the fact that $(M, w, \operatorname{chn})$ annotated-satisfies $x$, is is not too hard to see that $(M, w, \operatorname{chn})$ also annotated-satisfies $y$. Since $x$ is up-to-date and by definition of det-sts- $\beta$, we have alt $y \subseteq$ alt $_{x}$. Together with Prop. 18(vii) and the assumption that $(M, v, w, \operatorname{chn})$ realises $x$, this implies that $(M, v, w, \operatorname{chn})$ realises $y$.

If $\varphi \notin \mathrm{Ev}$, let $y$ be a child of $x$ such that $M, w \Vdash \Gamma_{y}$. The existence of $y$ follows from $M, w \Vdash \Gamma_{x}$ and Prop. 11. Furthermore, let $\varphi_{1}, \ldots, \varphi_{k}(1 \leq k \leq 2)$ be all formulae which were actually added to $\Gamma_{x}$ to create $\Gamma_{y}$ in expand. If $\varphi_{i}(1 \leq k \leq 2)$ is an eventuality, we have $\operatorname{ann}_{y}\left(\varphi_{i}\right)=\perp$ because of $\operatorname{ann}_{x}=\operatorname{ann}_{y}$ and $\varphi_{i} \notin \Gamma_{x}$. We can then conclude that $\left(M, w, \mathrm{chn}^{\prime}\right)$ annotated-satisfies $y$ for some extension $\mathrm{chn}^{\prime}$ of chn by repeatedly applying Prop. 30(i). Since $x$ is up-to-date and by definition of det-sts- $\beta$, we have alt $y \subseteq$ alt $_{x}$. Together with Prop. 18(vii) and the assumption that $(M, v, w, \operatorname{chn})$ realises $x$, this implies that $\left(M, v, w, \operatorname{chn}^{\prime}\right)$ realises $y$.

(ii): It is easy to see that $\sigma_{\geq 1}$ is a model chain for $\psi$ and that we have $w R_{b} v^{\prime}$ and $M, v^{\prime} \Vdash \psi$. By construction in expand, we have $\operatorname{ann}_{y}=\operatorname{ann}^{\perp}$ and $\Gamma_{y}=$ $\{\psi\} \cup \Gamma$ for some $\Gamma \subseteq$ Fml such that $[l] \Gamma \subseteq \Gamma_{x}$. Since $(M, w)$ realises $x$, we have $M, w \Vdash[l] \Gamma$ and thus $M, v^{\prime} \Vdash \Gamma$. We can thus apply Prop. 30(ii) on $M$, $v^{\prime}, \psi, \Gamma$, and $\sigma_{\geq 1}$ and obtain a function $\operatorname{chn}^{\prime}: \mathrm{Ev} \rightarrow(\mathrm{Seq}(W \times \mathrm{Fml}))^{\perp}$ such that $\left(M, v^{\prime}, \operatorname{chn}^{\prime}\right)$ annotated-satisfies $\left(\Gamma \cup\{\psi\}\right.$, ann $\left.{ }^{\perp}\right)$, and hence $y$. By definition of det-sts- $\beta$ and the assumptions that $(M, w)$ realises $x$ and alt $y \subseteq \operatorname{alt}_{x}$, this implies that $\left(M, w, v^{\prime}, \operatorname{chn}^{\prime}\right)$ realises $y$ and $\operatorname{chn}^{\prime}(\psi)=\sigma_{\geq 1}$ if $\psi \in$ Ev. 
(iii): Assume that $(M, w)$ does not realise $y:=\operatorname{getChild}(x, \mathrm{cs})$. As $(M, v, w, \operatorname{chn})$ realises $x$, we have $M, w \Vdash \Gamma_{x}$. Furthermore, we have $\Gamma_{x}=\Gamma_{y}$ by construction in expand. Hence there must exist a $\Gamma \in \operatorname{alt}_{x}$ such that $M, w \Vdash\left(\Gamma_{y} \cup \Gamma\right)$. By construction in expand, we have $\Gamma_{y}=\Gamma_{x}$. Moreover, by construction in det-sts-spl and since $x$ is up-to-date, the node $x$ has a child $z$ which is not a state such that $\Gamma_{z}=\Gamma_{x} \cup \Gamma, \operatorname{ann}_{x}=\operatorname{ann}_{z}, \operatorname{pst}_{x}=\operatorname{pst}_{z}, \operatorname{ppr}_{x}=\operatorname{ppr}_{z}$, and alt $_{z} \subseteq$ alt $_{x}$. In particular, we have $\operatorname{ann}_{z}(\chi)=\perp$ for all eventualities in $\Gamma$. We can then conclude that $\left(M, w, \operatorname{chn}^{\prime}\right)$ annotated-satisfies $z$ for some extension $\mathrm{chn}^{\prime}$ of chn by repeatedly applying Prop. 30(i). Together with the assumption that $(M, v, w, \operatorname{chn})$ realises $x$, this implies that $\left(M, v, w, \operatorname{chn}^{\prime}\right)$ realises $z$.

Definition 33. For a node $x \in V$ and an eventuality $\varphi \in \Gamma_{y} \cap \mathrm{Ev}$, we define the property $\mathfrak{P}(x, \varphi)$ as " $x$ is open $\& \operatorname{prs}_{x}(\varphi) \neq \perp \& \forall(y, \psi) \in \operatorname{prs}_{x}(\varphi)$. $\left(\operatorname{sts}_{y} \neq\right.$ $\operatorname{closed}(\cdot) \Rightarrow \mathfrak{P}(y, \psi))$ ".

Note that although $\mathfrak{P}(x, \varphi)$ is recursive, it is well-defined because of Prop. 18(i).

Lemma 34. In this lemma, let $G$ be the graph at any time between two rule applications when Rule 3 is not applicable. Let $x \in V$ and $\varphi \in \Gamma_{x} \cap \operatorname{Ev}$ such that $\mathfrak{P}(x, \varphi)$ holds. Furthermore, let $y$ be a child of $x$ which is not closed. If $x$ is a state or a special node, we require additionally that $\varphi=\langle l\rangle \chi$ for some $l \in \mathrm{LPrg}$ and some $\chi \in \mathrm{Ev}$. If $x$ is a state, we also require that $y$ is the successor of $\varphi$. If $x$ is a state we define $\psi:=\chi$, else we define $\psi:=\varphi$. In all cases we have $\mathfrak{P}(y, \psi)$.

Proof. We assume that $\mathfrak{P}(y, \psi)$ does not hold and derive a contradiction. Because of Prop. 18(i), this means that there exists a finite sequence $\left(y_{0}, \psi_{0}\right), \ldots,\left(y_{n}, \psi_{n}\right)$ of node-eventuality pairs such that:

- $y_{0}=y, \psi_{0}=\psi$, and $y_{n}$ is not closed;

$-y_{i}$ is open and $\left(y_{i+1}, \psi_{i+1}\right) \in \operatorname{prs}_{y_{i}}\left(\psi_{i}\right) \neq \perp$ for all $0 \leq i<n$;

- either $y_{n}$ is not open or $\operatorname{prs}_{y_{n}}\left(\psi_{n}\right)=\perp$.

Note that $n=0$ is possible and that $\mathfrak{P}\left(y_{i}, \psi_{i}\right)$ does not hold for all $0 \leq i \leq n$.

Let $m \in 0, \ldots, n$ be the smallest index such that not $y_{m} \sqsubset x$; or $m:=n$ if $y_{i} \sqsubset x$ for all $0 \leq i \leq n$. Then the sequence $\left(y_{0}, \psi_{0}\right), \ldots,\left(y_{m}, \psi_{m}\right)$ has the following properties, most of them are inherited from above:

- $y_{0}=y, \psi_{0}=\psi$, and $y_{m}$ is not closed;

- $y_{i}$ is open and $\left(y_{i+1}, \psi_{i+1}\right) \in \operatorname{prs}_{y_{i}}\left(\psi_{i}\right) \neq \perp$ for all $0 \leq i<n$;

- either $y_{n}$ is not open or $\operatorname{prs}_{y_{n}}\left(\psi_{n}\right)=\perp$ or not $y_{m} \sqsubset x$.

The following arguments rely on the fact that all nodes are up-to-date and on the definitions of det-prs-child and-depending on whether $x$ is an $\alpha / \beta$ node, a state, or a special node-det-sts- $\beta$ or det-sts-state or det-sts-spl, respectively.

If $\operatorname{prs}_{y_{m}}\left(\psi_{m}\right)=\perp$, it is not too hard to see that det-prs-child $(x, \varphi)=\perp$ and hence $\operatorname{prs}_{x}(\varphi)=\perp$ which contradicts the assumption that $\mathfrak{P}(x, \varphi)$ holds.

If $y_{n}$ is not open or not $y_{m} \sqsubset x$, it is not too hard to see that we have $\left(y_{m}, \psi_{m}\right) \in \operatorname{det}-p r s-\operatorname{child}(x, \varphi)$. Together with the definition of filter, this 
implies $\left(y_{m}, \psi_{m}\right) \in \operatorname{prs}_{x}(\varphi) \cup\left\{(x, \chi) \mid \chi \in \Gamma_{x} \cap \operatorname{Ev}\right\}$. If $\left(y_{m}, \psi_{m}\right) \in \operatorname{prs}_{x}(\varphi) \cup$ $\{(x, \varphi)\}$ then $\mathfrak{P}\left(y_{m}, \psi_{m}\right)$ follows from $\mathfrak{P}(x, \varphi)$, but this contradicts the fact that $\mathfrak{P}\left(y_{m}, \psi_{m}\right)$ does not hold. If $\left(y_{m}, \psi_{m}\right)=(x, \chi)$ for some $\chi \in \Gamma_{x} \cap \mathrm{Ev}$ with $\chi \neq \varphi$, we have $\perp \neq \operatorname{prs}_{x}(\chi) \subseteq \operatorname{prs}_{x}(\varphi)$ by construction in filter and because of the transitivity in the function reach. Hence $\mathfrak{P}(x, \varphi)$ implies $\mathfrak{P}(x, \chi)=$ $\mathfrak{P}\left(y_{m}, \psi_{m}\right)$ which again is a contradiction.

Lemma 35. If a node in $G$ is closed then it is not realisable.

Proof. We proceed by induction on the order in which the nodes are closed. That is, when dealing with a node which has just become closed, we can assume that all other nodes in $G$ which are already closed are not realisable. We must consider all cases in the algorithm where a node can be closed.

If a node is closed because it contains an immediate contradiction, it cannot be realised by definition. If a node is closed because it contains an "at a world" cycle, it cannot be a state by construction. Furthermore, it is easy to see that nodes containing an "at a world" cycle cannot be annotated-satisfiable.

If an $\alpha$ - or a $\beta$-node or a special node $x$ is closed because all its children are closed then $x$ is not realisable because of the induction hypothesis on the children and Lemma 32(i) and (iii).

If a state $x \in V$ is closed because one of its children is closed, let $\langle l\rangle \varphi$ be the $\langle\mathrm{lp}\rangle$-formula in $x$ such that its successor $y$ is already closed and alt $_{x}=$ $\mathrm{alt}_{y}$. The claim then follows from the induction hypothesis on $y$, Prop. 27, and Lemma 32(ii). Note that a model chain can always be shortened such that it does not contain a pair twice.

The last and most interesting case is when a node $x_{\mathrm{i}} \in V$ is closed in Rule 4 because there exists an eventuality $\varphi_{\mathrm{i}} \in \Gamma_{x_{\mathrm{i}}} \cap$ Ev such that $\operatorname{prs}_{x_{\mathrm{i}}}\left(\varphi_{\mathrm{i}}\right)=\emptyset$. For the rest of the proof, we consider $G$ at that moment right before closing $x_{\mathrm{i}}$. Next we assume that $x_{\mathrm{i}}$ is realisable and derive a contradiction.

For a contradiction, we distinguish whether or not $x_{\mathrm{i}}$ is a state. If $x_{\mathrm{i}}$ is a state, let $M=(W, R, \mathcal{V})$ be a model and $w_{\mathrm{i}} \in W$ such that $\left(M, w_{\mathrm{i}}\right)$ realises $x_{\mathrm{i}}$. Furthermore, let $\sigma$ be a model chain for $\left(M, w_{\mathrm{i}}, \varphi_{\mathrm{i}}, \operatorname{ex}\left(\varphi_{\mathrm{i}}\right)\right)$ which exists due to Prop. 27. If $x_{\mathrm{i}}$ is an $\alpha$ - or a $\beta$-node or a special node, let $M=(W, R, \mathcal{V})$ be a model, $w_{\mathrm{i}}, v_{\mathrm{i}} \in W$, and $\operatorname{chn}_{\mathrm{i}}: \mathrm{Ev} \rightarrow(\operatorname{Seq}(W \times \mathrm{Fml}))^{\perp}$ a function such that $\left(M, v_{\mathrm{i}}, w_{\mathrm{i}}, \operatorname{chn}_{\mathrm{i}}\right)$ realises $x_{\mathrm{i}}$. Furthermore, let $\sigma:=\operatorname{chn}_{\mathrm{i}}\left(\varphi_{\mathrm{i}}\right)$, that is $\sigma$ is a model chain for $\left(M, w_{\mathrm{i}}, \varphi_{\mathrm{i}}, \operatorname{ex}\left(\varphi_{\mathrm{i}}\right)\right)$. In both cases, it is not too hard to see that we can assume without loss of generality that $\sigma_{i} \neq \sigma_{j}$ for all $0 \leq i, j<|\sigma|, i \neq j$.

We will inductively define an arbitrarily long sequence $\omega \in \operatorname{Seq}\left(V \times \mathbb{N}_{0}\right)$, initially starting with $\omega:=\left(x_{\mathrm{i}}, 0\right)$, such that the following invariant is maintained for all $0 \leq k<|\omega|$ :

Invariant: Let $(x, i):=\omega_{k} \in V \times \mathbb{N}_{0}$.

- if $k>0$ and $(y, j):=\omega_{k-1}$ then: $x$ is a child of $y$ and $i \geq j$, and if $z$ is a state then $i>j$;

- we have $0 \leq i<|\sigma|$, let $(w, \varphi):=\sigma_{i} \in W \times \mathrm{Fml}$;

$-\varphi \in \Gamma_{y} \cap \operatorname{Ev}$ and $\mathfrak{P}(x, \varphi)$ holds;

- if $x$ is a state then $(M, w)$ realises $x$ and $\varphi$ is a $\langle\operatorname{lp}\rangle$-formula; 
- if $x$ is an $\alpha$ - or a $\beta$-node or a special node, there exists a $v \in W$ and a function $\operatorname{chn}: \operatorname{Ev} \rightarrow(\operatorname{Seq}(W \times \mathrm{Fml}))^{\perp} \operatorname{such}$ that $\operatorname{chn}(\varphi)=\sigma_{\geq i}$ and $(M, v, w, \operatorname{chn})$ realises $x$.

It is not difficult to see that the initial sequence $\omega=\left(x_{\mathrm{i}}, 0\right)$ fulfils the invariant. Note in particular that if $x_{\mathrm{i}}$ is a state then $\varphi_{\mathrm{i}}$ is a $\langle\mathrm{lp}\rangle$-formula by construction in det-sts-state.

Before we describe the construction of $\omega$, we show how we can use $\omega$ to derive a contradiction. Because of Prop. 18(iii), we can make $\omega$ long enough so that it contains $|\sigma|+1$ (not necessarily different) states. Hence $\omega$ must contain an element $(x, j)$ with $j \geq|\sigma|$ due to the invariant; but this is not possible since the invariant also guarantees that the natural numbers in $\omega$ are strictly smaller than $|\sigma|$. Next we will show how to construct $\omega$.

Let $(x, i)$ be the last pair in $\omega$ as constructed so far and $(w, \varphi):=\sigma_{i}$. We distinguish whether $x$ is an $\alpha / \beta$-node or a state or a special node.

Case 1 ( $x$ is $\alpha / \beta$-node): Let $v \in W$ and chn $: \mathrm{Ev} \rightarrow(\operatorname{Seq}(W \times \mathrm{Fml}))^{\perp}$ be a function such that $\operatorname{chn}(\varphi)=\sigma_{\geq i}$ and $(M, v, w, \operatorname{chn})$ realises $x$. Using Lemma 32(i), we obtain a child $y$ of $x$ and an extension $\operatorname{chn}^{\prime}$ of chn such that $\left(M, v, w, \operatorname{chn}^{\prime}\right)$ realises $y$. In particular, we have $\operatorname{chn}^{\prime}(\varphi)=\sigma_{\geq i}$. Furthermore $y$ cannot be closed because of the induction hypothesis. We extend $\omega$ by $(y, i)$. It remains to show that property $\mathfrak{P}(y, \varphi)$ holds, but this is exactly what Lemma 34 does.

Case 2 ( $x$ is a state): Because of the invariant, we know that $\varphi$ is of the form $\langle l\rangle \psi \in \mathrm{Ev}$ for some $l \in \operatorname{LPrg}$ and some $\psi \in \operatorname{Ev}$. Let $v^{\prime} \in W$ such that $\left(v^{\prime}, \psi\right)=\operatorname{chn}(\langle l\rangle \psi)_{1}=\sigma_{i+1}$ and $y$ be the successor of $\langle l\rangle \psi$. By construction in det-sts-state and the fact that $x$ is open and up-to-date, we have alt $_{y} \subseteq$ alt $_{x}$. Using Lemma 32(ii) on $y$ and $\langle l\rangle \psi$ and $\sigma \geq i$, we obtain a function $\operatorname{chn}^{\prime}: \mathrm{Ev} \rightarrow(\operatorname{Seq}(W \times \mathrm{Fml}))^{\perp}$ such that $\left(M, w, v^{\prime}, \operatorname{chn}^{\prime}\right)$ realises $y$ and $\operatorname{chn}^{\prime}(\psi)=\sigma_{\geq i+1}$. In particular we have $i+1<|\sigma|$ since $\psi \in$ Ev. Furthermore $y$ cannot be closed, either because of $y=x_{\mathrm{i}}$ or because of the induction hypothesis. We extend $\omega$ by $(y, i+1)$. It remains to show that property $\mathfrak{P}(y, \psi)$ holds which is done in Lemma 34.

Case 3 ( $x$ is a special node): Let $v \in W$ and chn $: \operatorname{Ev} \rightarrow(\operatorname{Seq}(W \times \mathrm{Fml}))^{\perp}$ be a function such that $\operatorname{chn}(\varphi)=\sigma_{\geq i}$ and $(M, v, w, \operatorname{chn})$ realises $x$. Using Lemma 32(iii), either $(M, w)$ realises $y:=\operatorname{getChild}(x, \mathrm{cs})$ or there exists a child $y$ of $x$ and an extension $\operatorname{chn}^{\prime}$ of chn such that $y$ is not a state and $\left(M, v, w, \operatorname{chn}^{\prime}\right)$ realises $y$. In both cases $y$ cannot be closed because of the induction hypothesis.

If $\varphi$ is a not a $\langle\mathrm{lp}\rangle$-formula then we consider $\varphi^{\prime}:=\operatorname{ann}_{x}(\varphi)$ which must be defined as $x$ is a special node. We know $\varphi^{\prime} \in \operatorname{Ev}$ since we would have $\operatorname{prs}_{x}(\varphi)=\perp$ by construction in det-sts $\beta$ otherwise. As $(M, w, \operatorname{chn})$ annotated-satisfies $x$, we therefore have $\operatorname{chn}(\varphi)_{1}=\left(w, \varphi^{\prime}\right)$ and hence $\operatorname{chn}\left(\varphi^{\prime}\right)=\operatorname{chn}(\varphi)_{\geq 1}$. Due to the invariant we have $\operatorname{chn}(\varphi)=\sigma_{\geq i}$ and thus $\operatorname{chn}\left(\varphi^{\prime}\right)=\sigma_{\geq i+1}$ and in particular $i+$ $1<|\sigma|$. Furthermore we have $\operatorname{prs}_{x}(\varphi)=\operatorname{prs}_{x}\left(\varphi^{\prime}\right)$ by definition of det-sts-state and because of Prop. 18(ii). Thus $\mathfrak{P}\left(x, \varphi^{\prime}\right)$ holds since $\mathfrak{P}(x, \varphi)$ holds. If $\varphi^{\prime}$ is a not a $\langle\mathrm{lp}\rangle$-formula, we consider $\varphi^{\prime \prime}:=\operatorname{ann}_{x}\left(\varphi^{\prime}\right)$ and so on. Since $x$ is open, and hence $\mathrm{ann}_{x}$ is non-cyclic, we will eventually obtain a $\langle\mathrm{lp}\rangle$-formula $\langle l\rangle \psi \in \mathrm{Ev}$ for 
some $l \in \mathrm{LPrg}$ and some $\psi \in \mathrm{Fml}$ such that $\mathfrak{P}(x,\langle l\rangle \psi)$ holds and $\operatorname{chn}(\langle l\rangle \psi)=$ $\sigma_{\geq i+j}$ for some $j \in \mathbb{N}_{0}$ with $i+j<|\sigma|$. Since $\mathrm{chn}^{\prime}$, if it is needed, is an extension of chn, we also have $\operatorname{chn}^{\prime}(\langle l\rangle \psi)=\sigma_{\geq i+j}$. In particular, we have $\sigma_{\geq i+j}=(w,\langle l\rangle \psi)$. Note that $\varphi=\langle l\rangle \psi$, and thus $j=0$, is possible.

We extend $\omega$ by $(y, i+j)$. It remains to show that property $\mathfrak{P}(y,\langle l\rangle \psi)$ holds which is shown by Lemma 34 and the established fact that $\mathfrak{P}(x,\langle l\rangle \psi)$ holds.

Theorem 36. If $\mathrm{rt}$ is closed then $\phi$ is unsatisfiable.

Proof. Assume for a contradiction that $\phi$ is satisfiable. Since the dummy atomic program $d$ does not occur in $\phi$, it is not too hard to see that $\langle d\rangle \phi$ is also satisfiable. Hence there exists a model $M=(W, R, \mathcal{V})$ and a world $w \in W$ such that $M, w \Vdash$ $\langle d\rangle \phi$. Together with Prop. 18(viii) and the fact that $\Gamma_{\mathrm{rt}}=\{\langle d\rangle \phi\}$, this implies that $(M, w)$ realises $x$ which contradicts Lemma 35 . 